\title{
Functional geometry of auditory cortical resting state networks derived from intracranial electrophysiology
}

Matthew I. Banks ${ }^{1,2^{*}}$, Bryan M. Krause ${ }^{1}$, D. Graham Berger ${ }^{1}$, Declan I. Campbell ${ }^{1}$, Aaron D. Boes ${ }^{3}$, Joel E. Bruss ${ }^{3}$, Christopher K. Kovach ${ }^{4}$, Hiroto Kawasaki ${ }^{4}$, Mitchell Steinschneider ${ }^{5,6}$, Kirill V. Nourski ${ }^{4,7}$

\section{*Corresponding author:}

Matthew I. Banks, Ph.D.

Professor

Department of Anesthesiology

University of Wisconsin

1300 University Avenue, Room 4605

Madison, WI 53706

Tel.: (608)261-1143

E-mail: mibanks@wisc.edu

\section{Keywords:}

Functional connectivity, hierarchy, fMRI, diffusion map embedding, electrocorticography, iEEG,

ECOG

\section{Acknowledgements}

This work was supported by the National Institutes of Health (grant numbers R01-DC04290,

R01-GM109086, S100D025025, UL1-RR024979). We are grateful to Jess Banks, Alex Billig, Haiming Chen, Phillip Gander, Christopher Garcia, Matthew Howard, Ariane Rhone, and Matthew Sutterer for help with data collection, analysis, and comments on the manuscript. 


\section{Abstract}

37 Critical details remain unresolved about the organization of the human auditory cortical

38 hierarchy and its relationship to higher order brain networks. We investigated this organization

39 using diffusion map embedding (DME) applied to resting state intracranial

40 electroencephalography (iEEG) obtained in neurosurgical patients. DME was applied to

41 functional connectivity measured between regions of interest (ROIs). ROIs exhibited a

42 hierarchical organization, symmetric between the two hemispheres and robust to the choice of

43 iEEG frequency band, connectivity metric, and imaging modality. Tight clusters of canonical

44 auditory and prefrontal ROIs were maximally segregated in embedding space. Clusters

45 consistent with ventral and dorsal auditory processing streams were paralleled by a cluster

46 suggestive of a third stream linking auditory and limbic structures. Portions of anterior

47 temporal cortex were characterized as global hubs. This approach lays the foundation for

48 identifying network changes during active speech and language processing and elucidating

49 mechanisms underlying disorders of auditory processing. 


\section{Introduction}

51 Extensive research has contributed to our understanding of the organization of the human 52 auditory cortical hierarchy ${ }^{1,2}$, yet fundamental questions remain unresolved. These include identification of secondary, tertiary, and higher order regions, hindering elucidation of the neural basis of speech and language comprehension. For example, the anterior portion of the superior temporal gyrus (STGA) and planum polare (PP) are adjacent to auditory cortex on Heschl's gyrus, yet diverge from it functionally ${ }^{3,4}$. Conversely, the posterior insula (InsP) has response properties similar to those of core auditory cortex in the posteromedial portion of Heschl's gyrus (HGPM), yet is not considered a canonical auditory area ${ }^{5}$. Additionally, the upper and lower banks of the superior temporal sulcus (STSU, STSL) have distinct roles in speech and language processing ${ }^{6,7}$, yet their functional relationships with other auditory areas are unclear.

Questions remain regarding broader organizational features as well. While the auditory hierarchy is posited to be organized along two processing streams ${ }^{8-10}$, the specific brain regions involved and the functional relationships within each stream are vigorously debated ${ }^{11-13}$. Furthermore, communication between auditory cortex and hippocampus, amygdala, and anterior insula (InsA) ${ }^{14}$ - areas involved in auditory working memory and processing of emotional aspects of auditory information ${ }^{15-18}$ - suggests a third "limbic" auditory processing stream, complementary to the dorsal and ventral streams. Additionally, while hemispheric lateralization of speech and language processing is a widely accepted organizational feature ${ }^{19,20}$, the degree to which it shapes the auditory hierarchy and is reflected in hemisphere-specific connectivity profiles is unknown $n^{8,12,21-24}$.

This study examined the organization of the human auditory cortical hierarchy within the broader context of cortical networks using resting state (RS) intracranial electroencephalography (iEEG) in neurosurgical patients. Although previous studies have investigated connectivity within the auditory cortical hierarchy using magnetic resonance imaging $(\mathrm{MRI})^{6,25,26}$, iEEG offers superior spatio-temporal resolution and is free of methodological problems that affect MRI in key regions such as the anterior temporal lobe ${ }^{27,28}$. Functional connectivity was measured as gamma band $(30-70 \mathrm{~Hz})$ power envelope correlations ${ }^{29}$ between regions of interest (ROIs) based on an auditory-centric cortical parcellation scheme. Functional geometry of the network was defined based on the connectivity profiles of its constituent ROls using diffusion map embedding (DME) ${ }^{30,31}$. DME maps ROls into a Euclidean space where proximity of two ROIs reflects similarity between their connectivity profiles. DME has been previously applied to functional MRI (fMRI) data to demonstrate the hierarchical structure of canonical RS networks ${ }^{32}$. Here, we applied DME to iEEG data for the first time to gain new insights into the functional organization of human auditory cortical networks. 


\section{Results}

Intracranial electrodes densely sampled cortical structures involved in auditory processing in the temporal and parietal lobes, as well as prefrontal, sensorimotor, and other ROIs in 46 participants (a total of 6487 sites; Fig. 1, Supplementary Tables 1, 2). Electrode coverage was largely restricted to a single hemisphere in individual participants. On average, each participant contributed $141 \pm 54$ recording sites, representing $28 \pm 7.9$ ROIs (mean \pm standard deviation) (see example in Fig. 2a). DME was applied to pairwise functional connectivity computed between recording sites in each participant. The functional connectivity matrix was normalized and thresholded to yield a transition probability matrix $\mathbf{P}$ with an apparent community structure along the horizontal and vertical dimensions (Fig. 2b). DME reveals the functional geometry of the sampled cortical sites by mapping the data of $\mathbf{P}$ into a low-dimensional embedding space where proximity between nodes represents similarity in their connectivity to the rest of the network (Fig. 2c; see Supplementary Fig. 1 for additional views).

100 DME exhibited superior signal-to noise characteristics compared to direct analysis of functional connectivity in 40 out of 46 participants (Supplementary Fig. 2). Sites that belonged to specific ROIs distributed to specific locations in embedding space. For example, in Figure 2c, tight clusters of auditory cortical sites (red/orange/yellow) and sites in prefrontal cortex (blue) were maximally segregated along dimension 1 (see Fig. 1 and Supplementary Table 3 for the list of abbreviations). Other ROIs [e.g., posterior portion of middle temporal gyrus (MTGP)] had a more distributed representation, consistent with their functional heterogeneity. 
bioRxiv preprint doi: https://doi.org/10.1101/2022.02.06.479292; this version posted February 22, 2022. The copyright holder for this preprint (which was not certified by peer review) is the author/funder, who has granted bioRxiv a license to display the preprint in perpetuity. It is made available under aCC-BY-NC 4.0 International license.
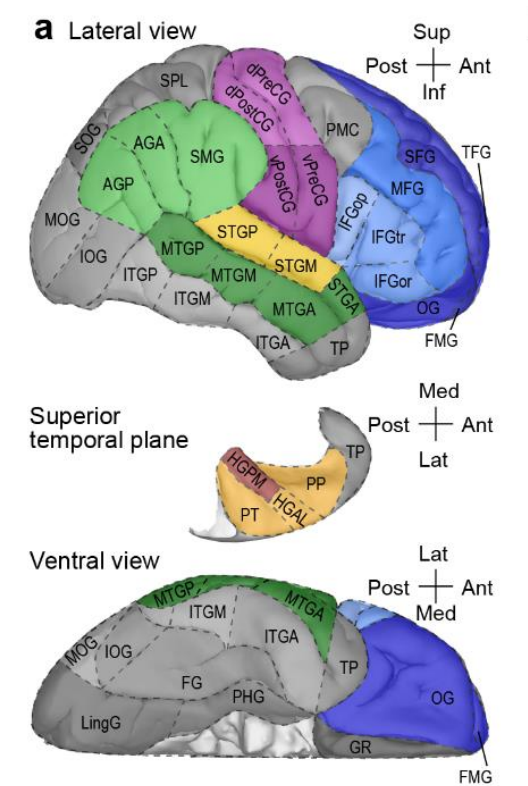

b Left hemisphere $\left(N_{\text {participants }}=24, n_{\text {stites }}=3278\right)$ Right hemisphere $\left(N_{\text {participants }}=22, n_{\text {sites }}=3209\right)$
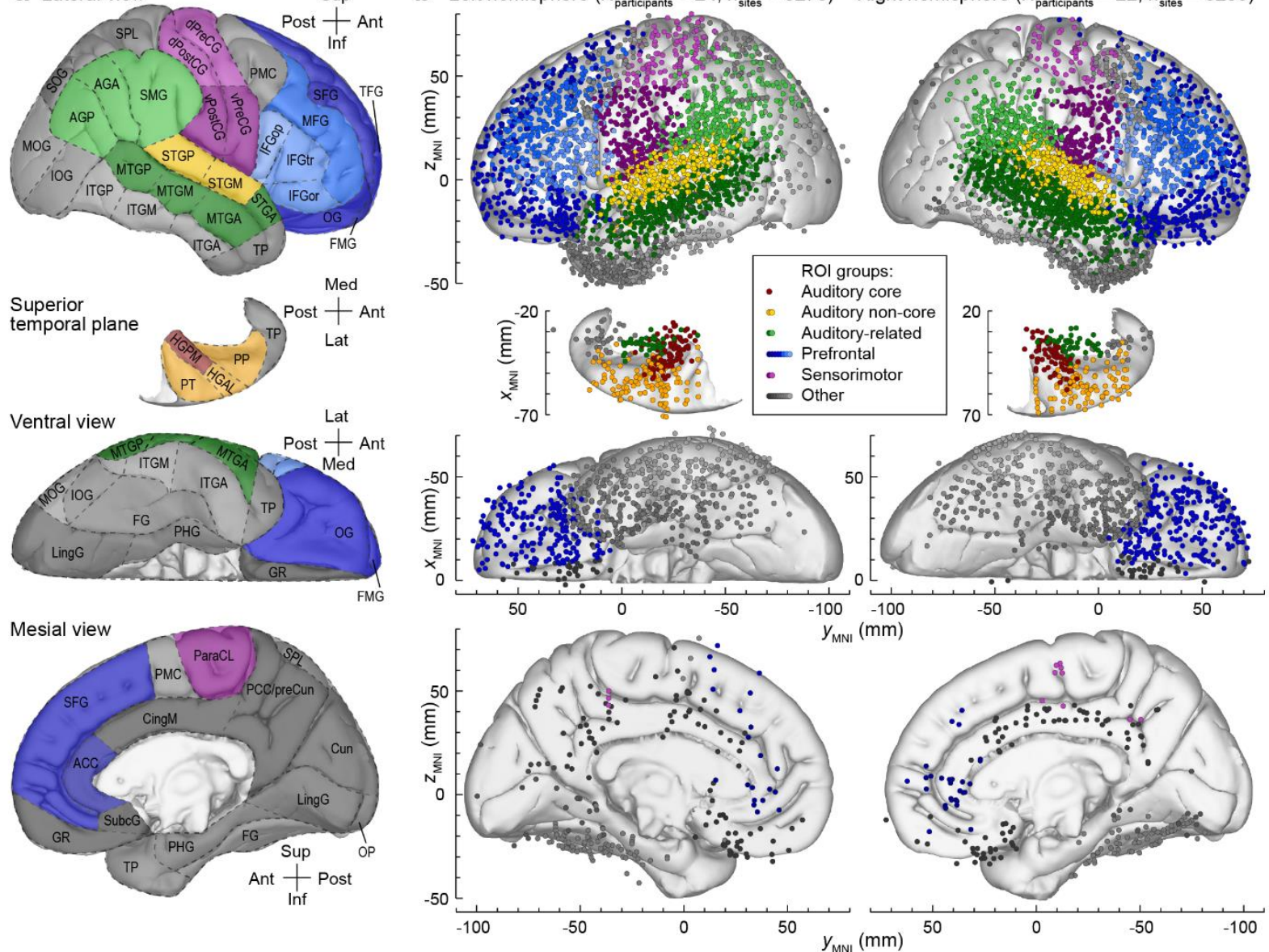

-100
$(\mathrm{~mm})$

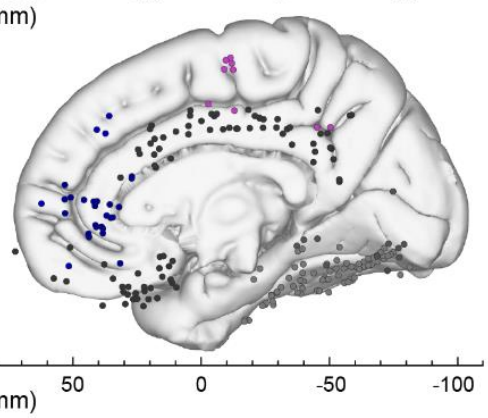

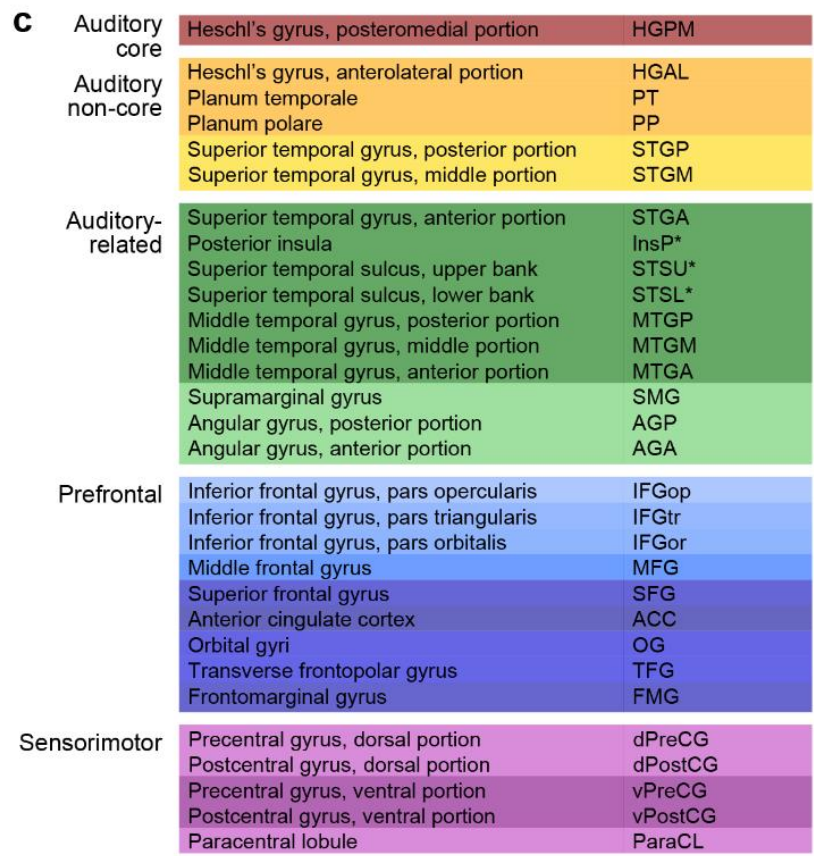

\begin{tabular}{|c|c|c|}
\hline \multirow{28}{*}{ Other } & Premotor cortex & PMC \\
\hline & Cingulate gyrus, middle portion & CingM \\
\hline & Cingulate gyrus, posterior portion / Precuneus & $\mathrm{PCC} /$ preCun \\
\hline & Parahippocampal gyrus & PHG \\
\hline & Fusiform gyrus & FG \\
\hline & Inferior temporal gyrus, posterior portion & ITGP \\
\hline & Inferior temporal gyrus, middle portion & ITGM \\
\hline & Inferior temporal gyrus, anterior portion & ITGA \\
\hline & Temporal pole & TP \\
\hline & Anterior insula & Ins $A^{*}$ \\
\hline & Frontal operculum & fOperc* ${ }^{*}$ \\
\hline & Parietal operculum & pOperc* \\
\hline & Gyrus rectus & GR \\
\hline & Subcallosal gyrus & SubcG \\
\hline & Superior parietal lobule / Intraparietal sulcus & SPL \\
\hline & Cuneus & Cun \\
\hline & Lingual gyrus & LingG \\
\hline & Occipital pole & OP \\
\hline & Superior occipital gyrus & SOG \\
\hline & Middle occipital gyrus & MOG \\
\hline & Inferior occipital gyrus & $10 G$ \\
\hline & Amygdala & Amyg* \\
\hline & Hippocampus & Hipp* \\
\hline & Putamen & Put* ${ }^{*}$ \\
\hline & Globus pallidus & $\mathrm{GP}^{\star}$ \\
\hline & Caudate nucleus & Caud* \\
\hline & Substantia innominata & Sublnn* \\
\hline & Ventral striatum & vStr* \\
\hline
\end{tabular}

*not pictured 
108 Figure 1. ROIs and electrode coverage in all 46 participants. a: ROI parcellation scheme. b: Locations of 109 recording sites, color-coded according to the ROI group, are plotted in Montreal Neurological Institute

110 (MNI) coordinate space and projected onto the Freesurfer average template brain for spatial reference.

111 Color shades represent different ROls within a group. Projections are shown on the lateral, top-down

112 (superior temporal plane), ventral and mesial views (top to bottom). Recording sites over orbital,

113 transverse frontopolar, inferior temporal gyrus and temporal pole are shown in both the lateral and the

114 ventral view. Sites in fusiform, lingual, parahippocampal gyrus and gyrus rectus are shown in both the

115 ventral and medial view. Sites in the frontal operculum $(n=21)$, parietal operculum $(n=15)$, amygdala

$116(n=73)$, hippocampus $(n=77)$, putamen $(n=13)$, globus pallidus $(n=1)$, caudate nucleus $(n=10)$,

117 substantia innominata $(n=5)$, and ventral striatum $(n=2)$ are not shown. See Supplementary Table 2 for

118 detailed information on electrode coverage. c: ROI groups, ROls and abbreviations used in the present

119 study. See Supplementary Table 3 for alphabetized list of abbreviations. 
bioRxiv preprint doi: https://doi.org/10.1101/2022.02.06.479292; this version posted February 22, 2022. The copyright holder for this preprint (which was not certified by peer review) is the author/funder, who has granted bioRxiv a license to display the preprint in perpetuity. It is made available under aCC-BY-NC 4.0 International license.

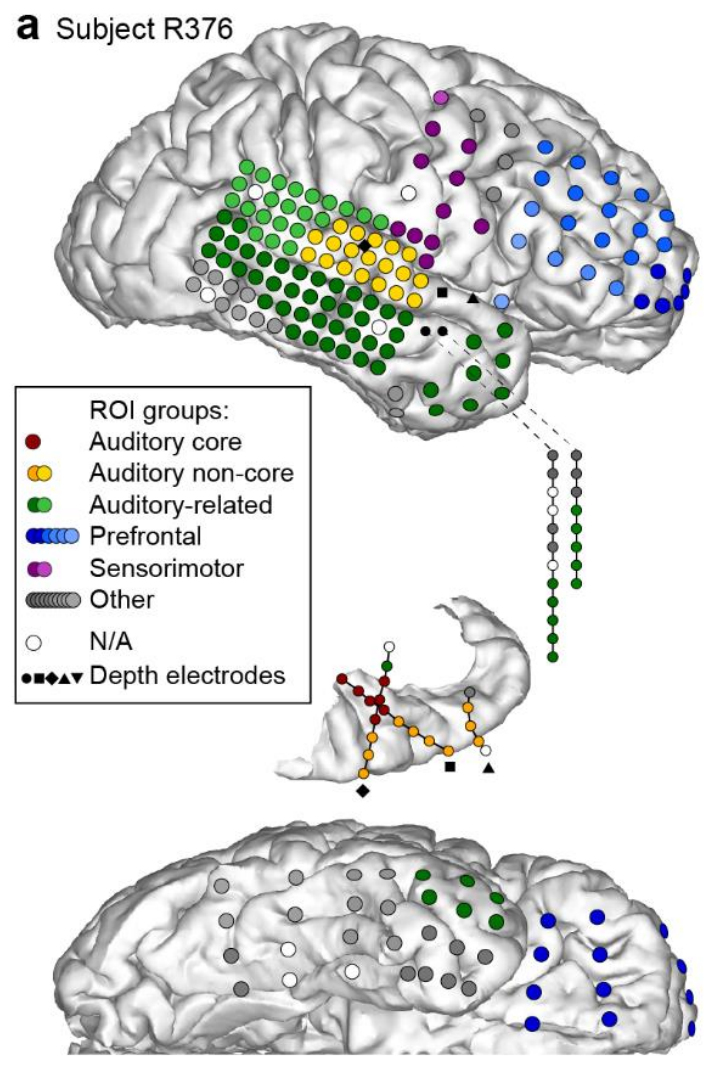

C $2 \mathrm{D}$ projections of data
in embedding space



b Transition probability matrix $\mathbf{P}$

Normalized relative connectivity:

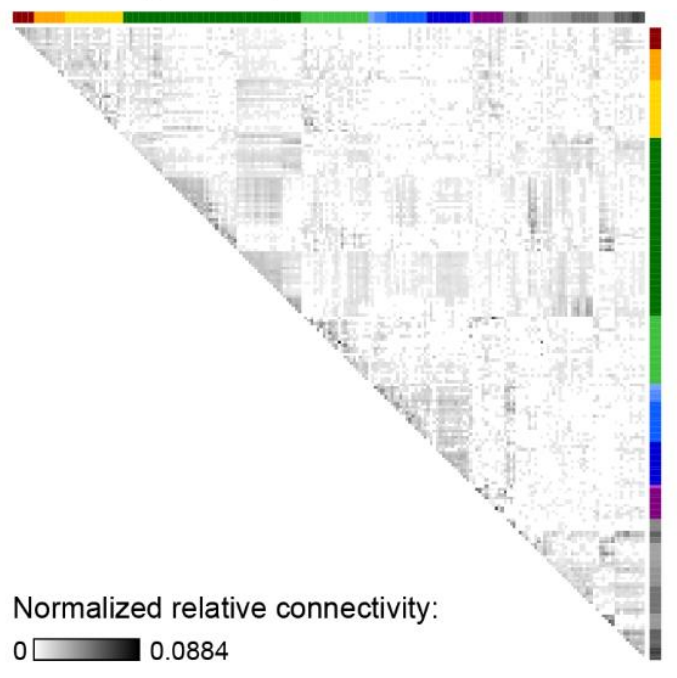

Dimension 1 SMG SMG




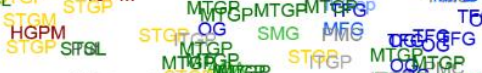

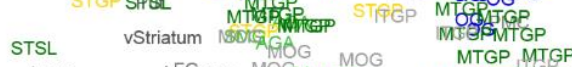





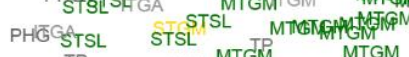

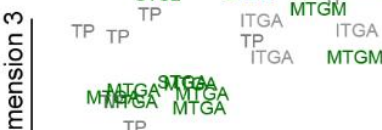

Dimension 1



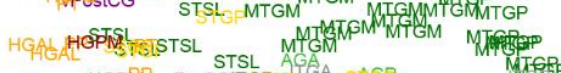

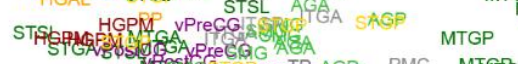

HGPM

MTSFGA

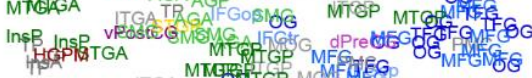

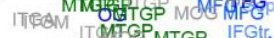

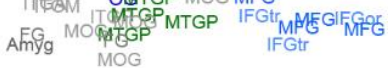

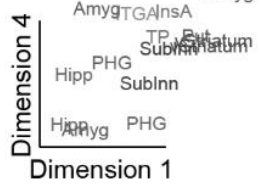

121 Figure 2. Functional geometry of cortical networks revealed by DME in a single participant (R376). a:

122 Electrode coverage. b: Normalized adjacency matrix. c: Data plotted in the 1st and 2nd, 1st and 3rd, and

123 1st and 4th dimensions of embedding space (top to bottom). Two points that are close in embedding

124 space are similarly connected to the rest of the network, and thus assumed to be functionally similar.

125 Scale bar: 0.03. 
127 To pool data across participants with variable electrode coverage, $\mathbf{P}$ matrices were computed at the ROI level and averaged across participants (Fig. 3a). The eigenvalue spectrum $\left|\lambda_{i}\right|$ of $\mathbf{P}$ featured an inflection point for i>4 (Fig. 3a, inset), indicating that the first four dimensions of embedding space accounted for the community structure of the data. The data are plotted in the first four dimensions of embedding space in Figure 3b (see also Supplementary Fig. 3 and Supplementary Movies 1 and 2), providing a graphical representation of the functional geometry of all sampled brain regions. Functionally related ROIs clustered together, and these clusters segregated within embedding space. For example, auditory cortical and prefrontal ROls were at opposite ends of dimension 1, and parietal and limbic ROIs were at opposite ends of dimension 2. By contrast, some ROIs [e.g., STGA, anterior and middle portions of middle temporal gyrus (MTGA, MTGM)] were situated in the interior of the data cloud.

The connectivity metric employed here discards components exactly in phase between two brain regions, mitigating the influence of volume conduction ${ }^{29}$. However, brain areas that are anatomically close to each other are often densely interconnected ${ }^{33-37}$. Thus, anatomical proximity can contribute to the observed functional geometry. Auditory cortical ROls followed this rule and clustered together with one notable exception. PP, located immediately anterior to anterolateral Heschl's gyrus (HGAL), segregated from the rest of auditory cortical ROIs along dimension 2 in embedding space (Fig. 3b, upper panel, lower left corner). Separation in embedding space was also observed along specific dimensions between other anatomically adjacent ROIs, including STGA and STGM, temporal pole (TP) and the rest of the anterior temporal lobe (ATL), and InsA and InsP. Anatomical proximity explained $15 \%$ of the variance in embedding distance (mean adjusted $r^{2}=0.15$ for regressions between anatomical and embedding Euclidean distance, calculated separately for each ROI). Thus, the embedding representation elucidates organizational features of ROls beyond anatomical proximity.

151 The grouping of canonical auditory ROIs is apparent in Figure 3b, as PT, HGAL, and middle and 152 posterior portions of the superior temporal gyrus (STGM, STGP) were all close to HGPM in 153 embedding space. Interestingly, so was STSU, which was significantly closer to auditory cortex 154 in embedding space compared to STSL $(p=0.002)$. This separation between STSL and STSU is 155 consistent with differences in their response properties reported recently ${ }^{7}$. Particularly, 156 responses in STSL, but not STSU, were predictive of performance in a semantic categorization 157 task. This suggests that in embedding space, STSL would be closer to regions involved in 158 semantic processing compared to STSU. Indeed, a permutation analysis revealed that STSL was 159 closer to ROIs reported to contribute to semantic processing [inferior frontal gyrus (IFG) pars 160 operculum/triangularis/orbitalis (IFGop, IFGtri, IFGor), TP, STGA, MTGA, MTGP, anterior and 161 posterior portions of inferior temporal gyrus (ITGA, ITGP), anterior and posterior angular gyrus 162 (AGA, AGP), supramarginal gyrus (SMG) $]^{38-40}$ compared to STSU $(p=0.010)$. 

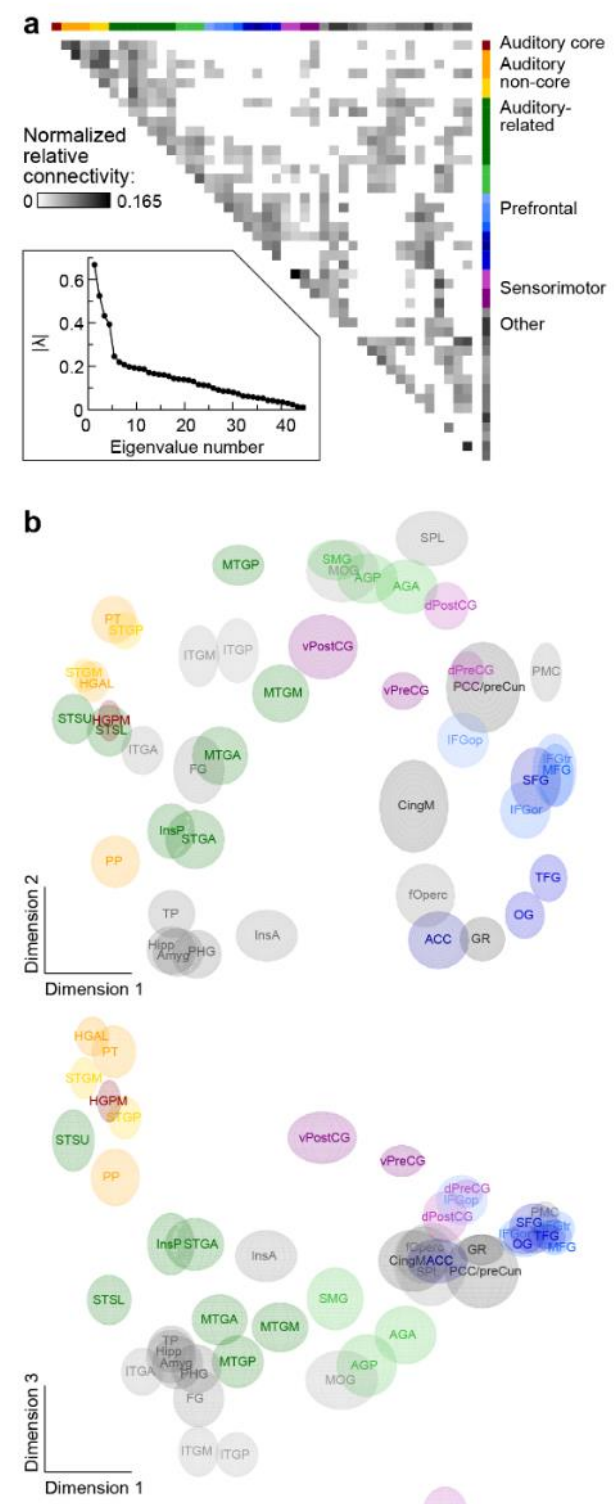

164 Figure 3. Summary of functional geometry of cortical networks via DME. a: Average normalized 165 adjacency matrix. Inset: Eigenvalue spectrum. b: Data plotted in the 1st and 2nd, 1st and 3rd, and 1st 166 and 4th dimensions of embedding space (top to bottom). Variance estimates on the locations of each 167 ROI in embedding space were obtained via bootstrapping and are represented by the size of the 168 ellipsoid for each ROI. Scale bar: 0.1. 
InsP responds robustly to acoustic stimuli ${ }^{5}$, suggesting that a portion of this area could be considered an auditory region ${ }^{41}$. It can track relatively fast $(>100 \mathrm{~Hz})$ temporal modulations, similar to $\mathrm{HGPM}^{5,42}$, possibly due to direct inputs from the auditory thalamus. However, InsP was functionally segregated from HGPM and was situated between auditory and limbic ROIs, consistent with the broader role of InsP in polysensory exteroceptive processing and interoception $^{43,44}$.

Figure $3 \mathrm{~b}$ also characterizes the temporal and parietal ROIs outside auditory cortex that are nonetheless part of the extended auditory network, including components of the dorsal and ventral processing streams. These 'auditory-related' ROls (shades of green in Fig. 3b), were distributed along a considerable extent of all four dimensions, consistent with functional heterogeneity of these regions and their involvement in multimodal integration ${ }^{45}$.

So far, the results of analyses have been presented at a single spatial scale $(t=1)$, which emphasized local network structure. Analysis of data at a coarser spatial resolution $(t=5)$ showed that the dominant structure in the data was governed by dimension 1 , with auditory ROIs at one end and prefrontal ROIs at the other (Supplementary Fig. 4). This indicates that the functional distance between these two groups of ROls is the most prominent of all organizational features in the data.

\section{Hierarchical clustering of ROIs}

Hierarchical clustering applied to the first four dimensions of the embedded data revealed segregation of the ROIs into several well-delineated clusters (Fig. 4). Auditory cortical ROIs (excluding PP) formed an 'Auditory' cluster with STSU. Another major cluster (labeled 'Limbic') included ROIs traditionally considered part of the limbic system [parahippocampal gyrus (PHG), amygdala and hippocampus], ATL ROIs (TP, STGA, PP), and the insula. ROls typically considered part of the ventral and dorsal auditory streams segregated into two clusters. Additional clusters included ROls involved in executive and sensorimotor functions (labeled 'Executive' and 'Action', respectively in Fig. 4). Thus, the hierarchical clustering analysis revealed a segregation of ROIs in embedding space that aligned with known functional differentiation of brain regions. This analysis expands our understanding of functional relationships within the auditory cortical hierarchy (e.g., clustering of STSU with canonical auditory ROIs and segregation of PP and InsP from HGPM) and between auditory and higher order cortical areas (e.g., the proximity of 'Auditory' and 'Limbic' clusters). 
bioRxiv preprint doi: https://doi.org/10.1101/2022.02.06.479292; this version posted February 22. 2022. The copyright holder for this preprint (which was not certified by peer review) is the author/funder, who has granted bioRxiv a license to display the preprint in perpetuity. It is made available under aCC-BY-NC 4.0 International license.



202 Figure 4. Hierarchical clustering of embedding data. Dendrogram showing linkages between ROI groups 203 identified using agglomerative clustering. 
205 Identification of 'global hubs' within brain networks is critical for understanding their 206 topology ${ }^{46}$. These nodes integrate and regulate information flow in the network by virtue of 207 their centrality and strong connectivity. DME can identify global hubs, as the closer an ROI is to 208 the center of the data cloud in embedding space, the more equal is its connectivity to the rest 209 of the network. This is illustrated in Figure 5a, which depicts a simulated network of five ROls, 210 with one serving as a global hub (Fig. 5a, left panel, green). The network structure can also be 211 represented as an adjacency matrix, wherein the hub ROI has strong connectivity with other 212 ROIs (Fig. 5a, middle panel). In embedding space, this ROI occupies a central location, with the 213 other four serving as spokes, i.e., nodes that interact with each other through the central hub 214 (Fig. 5a, right panel).

215 However, a node's proximity to the center of the data cloud reflects the homogeneity of its 216 connectivity to the rest of the network. Thus, a node can appear at a central location if it is 217 weakly but consistently connected to all other nodes. By contrast, a node strongly connected to 218 a small number of nodes ('local hub') can have high mean connectivity but still be distant from 219 the center of the data cloud. Combining distance from the center with mean connectivity (Fig. 5b) allows for classification of ROIs as global versus local hubs and for distinguishing hubs from spokes and central nodes that have relatively weak overall connectivity ('bridge'). ATL ROIs

222 (MTGA, STGA, but not TP) and MTGM lie in the upper left quadrant of the plot $>2$ standard 223 deviations from the center of the data cloud (outer dashed ellipse) and appear as global hubs. 224 ITGA, posterior cingulate/precuneus (PCC/preCun), and STSL also exhibited hub-like properties, 225 i.e., were located in the upper left quadrant of Figure 5b.

226 STSU and orbitofrontal gyrus (OG) tended toward high connectivity but were located at a 227 greater distance from the center and can thus be considered local hubs. Primary sensory and motor regions and canonical auditory cortex (except PP) tended to behave as spokes, occupying the lower right quadrant of the graph. Finally, the ROIs in the lower left quadrant can be considered bridges between isolated clusters of ROIs, characterized by more selective connectivity patterns. Thus, DME can identify topological features critical to information flow

232 within cortical networks. 

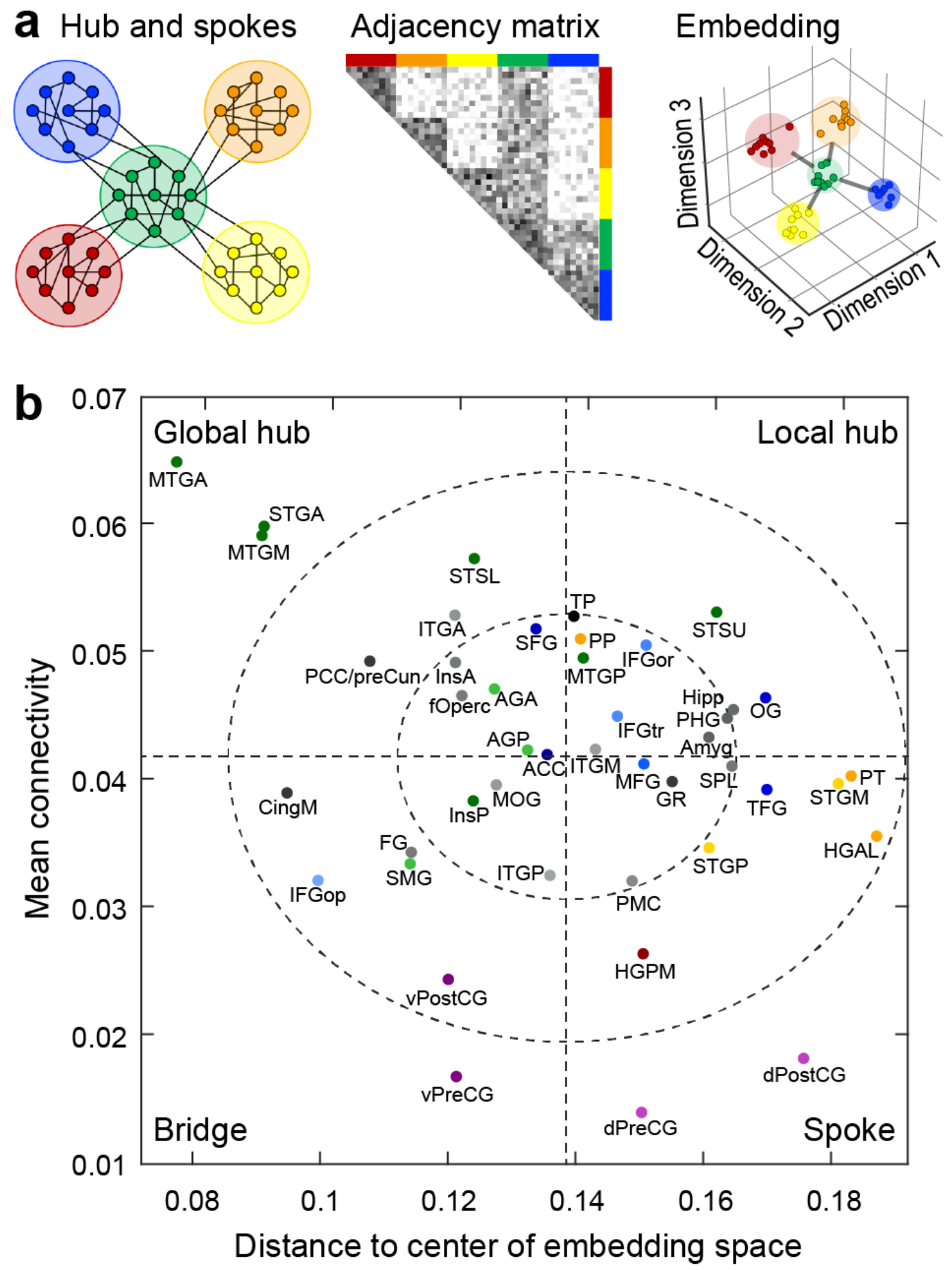

Figure 5. Identification of network hubs. a: Schematic example illustrating the central positioning of global hubs in embedding space. $\mathbf{b}$ : ROIs from average embedding are plotted according to their mean connectivity to the rest of the network versus their distance to the centroid of the data cloud in the first four dimensions of embedding space. Dashed lines denote across-ROI means. Dashed ellipses represent 
240

241

242

243

244

245

246

247

248

249

250

251

252

253

254

255

256

257

258

259

260

261

262

263

Speech and language networks are lateralized in the human brain, with nearly all right-handed and most left-handed individuals left hemisphere language-dominant ${ }^{47}$. However, both hemispheres are activated during speech processing $9,22,48,49$, and the extent to which lateralization is reflected in asymmetries in the organization of auditory networks is unclear. We addressed this issue by comparing the geometry of cortical networks derived from participants with electrode coverage in the language-dominant $(N=22)$ versus non-dominant $(N=21)$ hemisphere. ROls exhibited a symmetric functional organization in embedding space (Supplementary Fig. 5). Pairwise inter-ROI distances in embedding space, calculated separately for dominant versus non-dominant hemisphere, were highly correlated $(r=0.77)$, with no obvious outliers (Fig. 6, left panel). Furthermore, this was true specifically for ROls involved in speech and language comprehension and production [PT, PP, STSL, STGP, STGM, STGA, SMG, AGA, premotor cortex (PMC), precentral gyrus (PreCG), IFGop, IFGtr] $]^{12,50,51}$ ( $r=0.71$; Fig. 6, right panel). Permutation analysis indicated that neither the positions of ROIs in embedding space nor the pairwise distances between ROls were significantly different between dominant and non-dominant hemispheres (all $p$-values $>0.05$ ). Thus, hemispheric asymmetry of functional organization of speech and language networks was not detectable in RS connectivity.

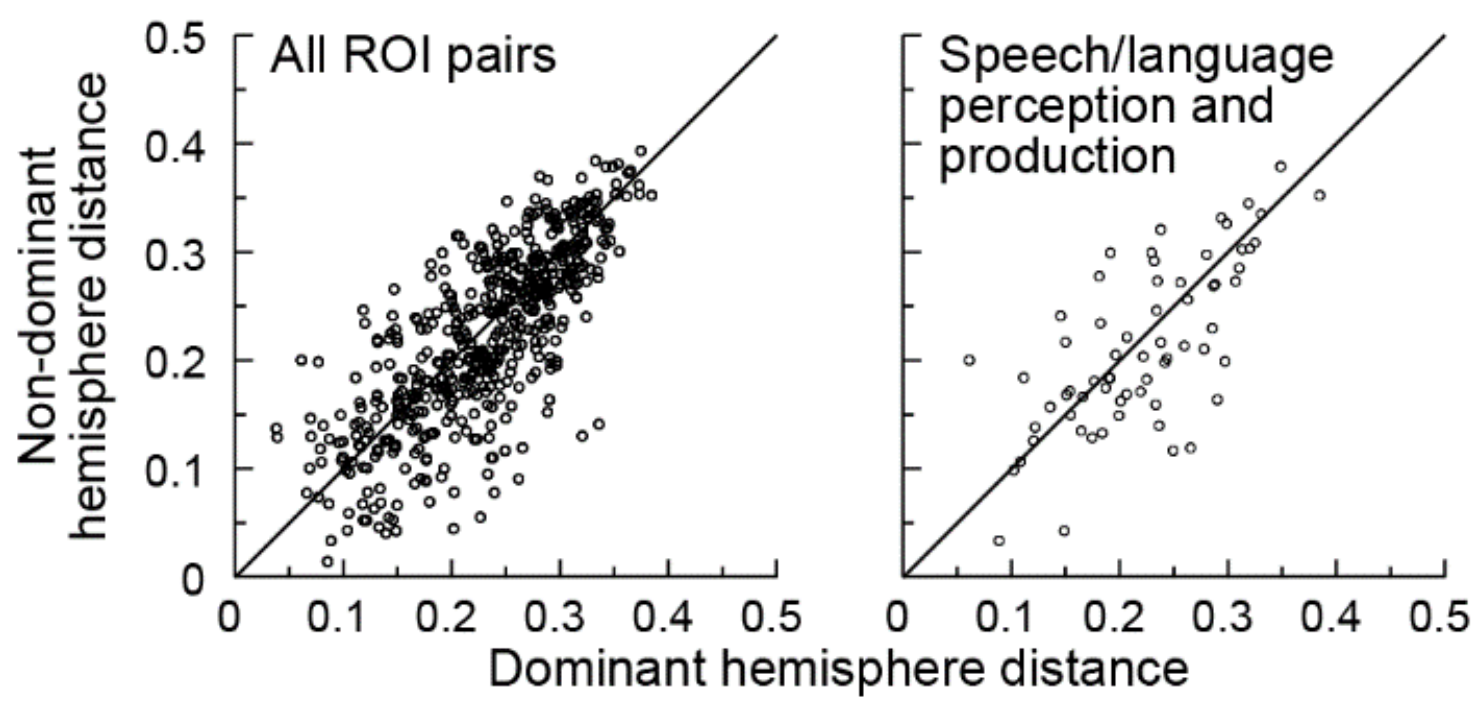

Figure 6. RS connectivity is symmetric between hemispheres. Inter-ROI distances in embedding space for non-dominant versus dominant hemisphere participants. Pairwise distances between all ROIs and between ROls involved in speech and language perception and production (PT, PP, STSL, STGP, STGM, STGA, SMG, AGA, PMC, PreCG, IFGop, IFGtr) are shown in the left and right panel, respectively. Note that after splitting the data into the two subsets (dominant and non-dominant) STSU did not meet the inclusion criteria for analysis presented in the right panel (see Methods, Supplementary Table 2). 
Stability of functional geometry across frequency band and connectivity measures

Embeddings presented so far have been derived from gamma-band power envelope correlations. Other bands (theta, alpha, beta, high gamma) and a different measure (debiased weighted phase lag index, $\mathrm{WPLI}^{52}$ ) produced similar embeddings. For power envelope correlations, inter-ROI distances were highly similar for adjacent bands ( $r \geq 0.83$ ), and even for non-adjacent bands ( $r \geq 0.72$; Supplementary Fig. 6a). For wPLI, correlations were only slightly weaker (0.59-0.79). Correlations were strong even across the two connectivity measures (0.580.78). Thus, DME identified overall, rather than band- or measure-specific, organizational features of cortical networks.

However, a particular band or measure might be preferred if it produced narrower estimation margins in the functional geometry. An overall relative uncertainty was calculated as the variation of ROI position across bootstrapped samples relative to the average distance to other ROIs. Relative uncertainty was similar across bands and measures but was lowest for power envelope correlations in beta, gamma, and high gamma bands (Supplementary Fig. 6b). These analyses suggest that gamma power envelope correlations are a robust measure for exploring functional geometry.

\section{Comparison to embeddings derived from RS-fMRI data}

Intracranial recordings sample the brain non-uniformly and sparsely as dictated by clinical considerations. To examine the impact of this sampling, DME was applied to RS-fMRI data available in a subset of ten participants. We first verified the consistency of functional geometry derived from the two modalities in the same participants (Fig. 7). Connectivity matrices were constructed based on RS-fMRI data from voxels located at iEEG recording sites and grouped into the same ROIs as in Figure 1. The iEEG and $\mathrm{FMRI}$ embeddings averaged across participants were qualitatively similar (Fig. 7a, b), and the overall organization derived from this subset was consistent with that observed in the full iEEG dataset (cf. Fig. 3b). Inter-ROI distances in the $\mathrm{fMRI}$ and iEEG embedding spaces were strongly correlated (Fig. 7), with highest correlations for beta-, gamma- and high gamma-band envelopes ( $r>0.5$; Fig. 7d, line and symbols). Similar results were observed at the individual participant level as well, albeit with smaller $r$ values (Fig. 7d, box and whiskers plot).

The analysis presented in Figure 7 indicates that $\mathrm{fMRI}$ data can be used to address two questions regarding the effects of limited, non-uniform sampling. The first question is the effect of non-uniformly sampling only a subset of brain regions. We used a standard parcellation scheme developed for fMRI data (Schaefer-Yeo 400 ROIs; ${ }^{53}$ ) rather than the iEEG parcellation scheme introduced in Figure 1. For each participant, embeddings were derived from RS-fMRI connectivity matrices computed from all cortical ROls ("Full fMRI", Fig. 8a, leftmost column). A subset of these ROIs ["Full fMRI (iEEG subset)", Fig. 8a, 2nd column] were retained for comparison to embeddings computed from the $\mathrm{fMRI}$ data corresponding to the ROIs sampled 
302 by iEEG ["Partial fMRI (ROI level)", Fig. 8a, 3rd column]. The two embeddings were compared by

303 computing the correlation between inter-ROI distances in the respective embedding spaces

304 (Fig. 8b). Although the scale of the embeddings was different for the full fMRI versus partial

$305 \mathrm{fMRI}$ data (because the number of dimensions was different), the two were highly correlated ( $r$

$306=0.94$; Fig. 8c). Thus, embeddings constructed from the portion of the brain sampled by iEEG

307 were nearly identical to embeddings derived from the whole brain.

308 The second question is the effect of representing an entire ROI by sparse sampling with a 309 limited number of electrodes. To address this question, embeddings were computed from the 310 averages across entire ROIs in each participant ["Partial fMRI (ROI level)", Fig. 8a, 3rd column] 311 and from averages of the fMRI voxels located at iEEG recording sites ["Partial fMRI (site level)", 312 Fig. 8a, rightmost column]. ROI- and site-level embedding distances were strongly correlated ( $r$ $313>0.5$; Fig. 8c). Thus, sparse sampling within an ROI had a greater impact on estimates of 314 functional geometry than limited sampling of the complete set of ROls. Overall, ROls were 315 faithfully represented in embedding space even when DME was based on a small number of 316 locations within ROIs. Taken together, these results indicate broad consistency between 317 functional organization derived from iEEG and $\mathrm{FMRI}$ and the robustness of this approach to 318 sparse sampling afforded by iEEG recordings. 

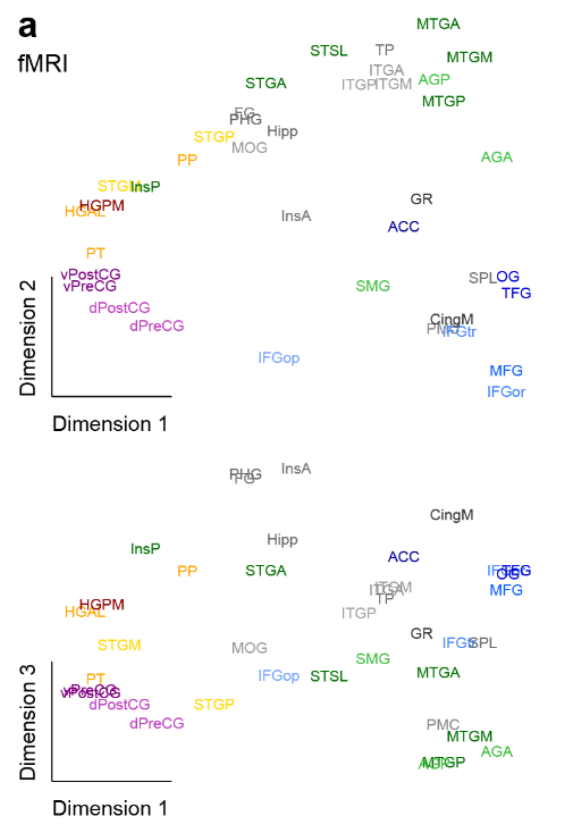

ACC

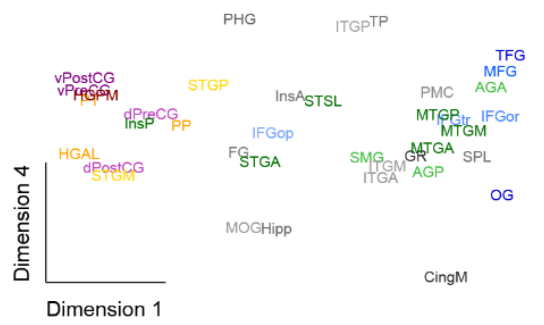

b

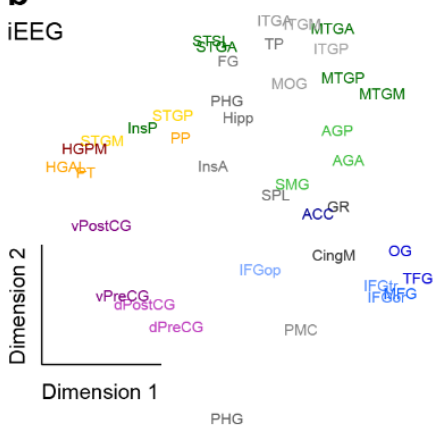

PHG

InsA ${ }_{\text {Hipp }}$
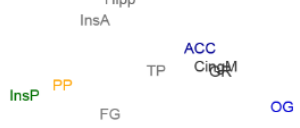

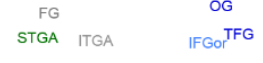

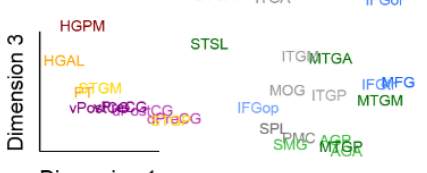

Dimension 1

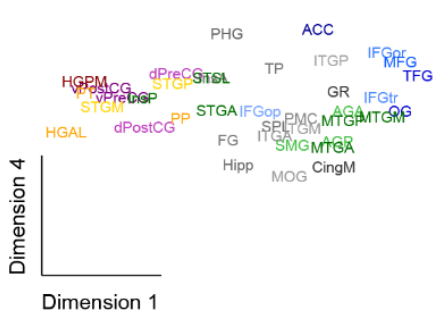

C Pairwise distance



d

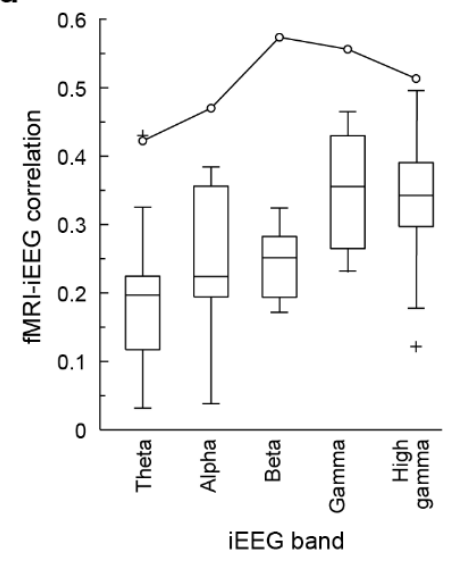

Figure 7. Comparison of iEEG and fMRI connectivity data in embedding space. a: Participant-averaged embeddings for iEEG (gamma band power envelope correlations). b: Participant-averaged embeddings

322 for fMRI. Scale bar: 0.1. c: Inter-ROI embedding distances computed from the data in a and b. d:

323 Summary of distance correlations at each frequency band for participant-averaged data (line and symbols) and single participants (box and whiskers). $t=1$ for all embeddings. 

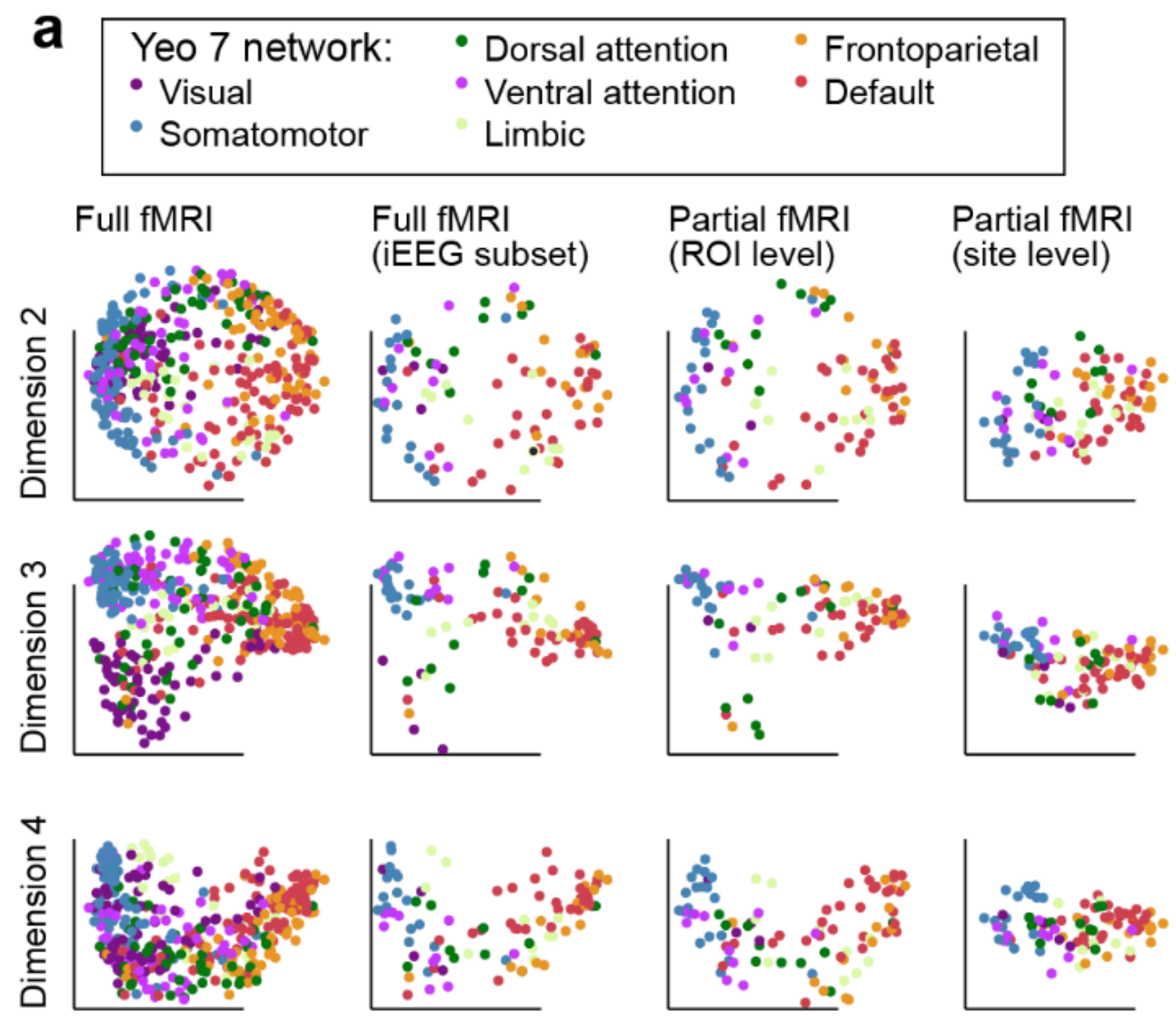

Dimension 1
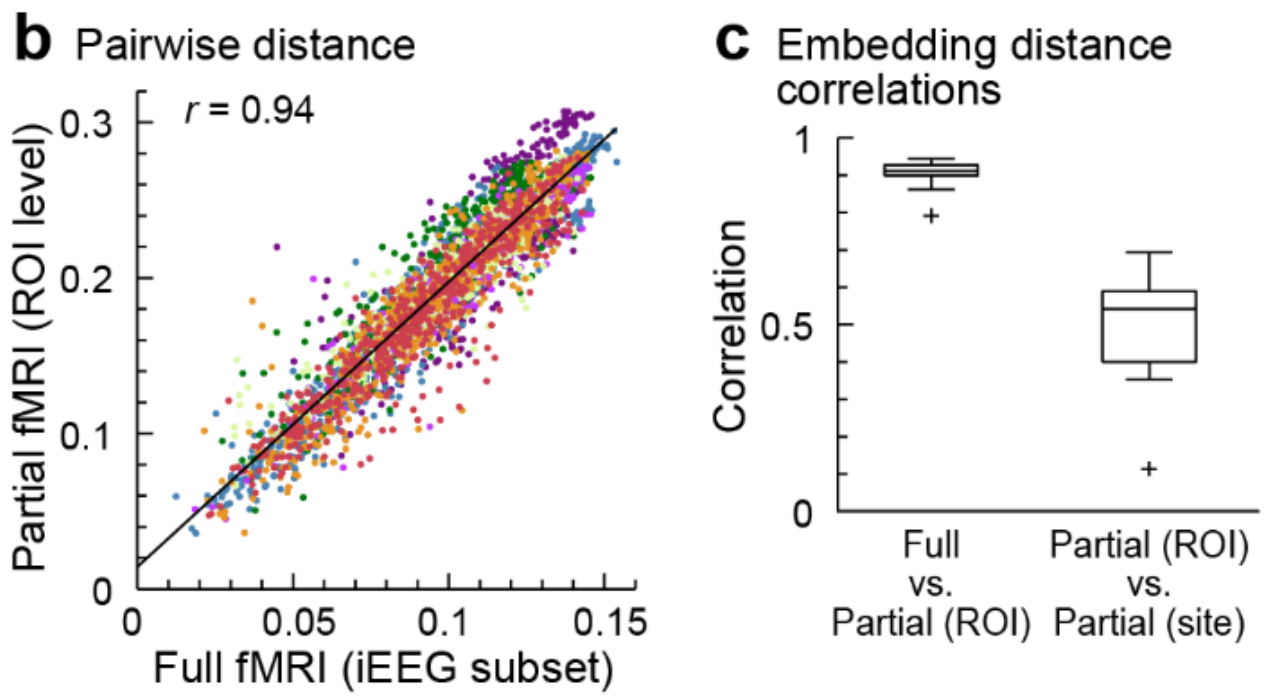

Figure 8. Effect of constructing embeddings from only a subset of ROIs in the brain. Scale bar: 0.1 (full 


\section{Discussion}

\section{Organization of auditory cortical networks}

330

331

332

333

334

335

336

337

338

339

340

341

342

343

344

345

346

347

348

349

350

351

352

353

354

355

356

357

358

359

360

We have shown that DME applied to iEEG data can be used to characterize the organization of the human auditory cortical hierarchy. Previous work in macaque has defined over a dozen auditory cortical fields based on cytoarchitectonics, connectivity, and response properties ${ }^{54}$. By contrast, there is no consensus on how auditory cortex is organized in humans, with multiple candidate parcellations based on cytoarchitectonics, tonotopy or myeloarchitecture ${ }^{55-58}$. Our results contribute to this body of knowledge by showing that superior temporal ROIs including core auditory cortex (HGPM) and putative auditory belt and parabelt areas (PT, HGAL, STGP, $\mathrm{STGM}^{55,58}$ ) cluster together in embedding space, indicating functional similarity. We could also discern hierarchical relationships within this cluster by combining embedding analysis with mean connectivity. Specifically, despite the proximity of HGPM to other auditory ROIs in embedding space (Fig. 3b), it exhibited weaker mean connectivity, suggesting that it functions as a spoke rather than a local hub (Fig. 5b). This is consistent with the position of HGPM at the lowest level of the auditory cortical hierarchy. STGP had an intermediate position between HGPM and HGAL/PT/STGM in terms of its eccentricity and mean connectivity. This suggests that STGP is the locus of relatively early non-core auditory cortex ${ }^{59,60}$, occupying a low-level position in the hierarchy ${ }^{61}$.

\section{Functional differentiation between STSU and STSL}

The superior temporal sulcus is a critical node in speech and language networks linking canonical auditory cortex with higher order temporal, parietal, and frontal areas ${ }^{6,48,50,62-64}$. Previous studies have shown that STSU and STSL differ in cytoarchitecture ${ }^{65}$ and have distinct responses to speech ${ }^{23,66-68}$. A recent iEEG study demonstrated enhanced, shorter-latency, responses to speech syllables in STSU compared to STSL ${ }^{7}$. STSU is traditionally not considered part of canonical auditory cortex (but see ${ }^{56}$ ), yet it clustered with auditory cortical ROIs. STSL, by contrast, was closer in embedding space to semantic ROls. This is consistent with iEEG evidence that responses in STSL, but not STSU, correlated with performance on a semantic categorization task ${ }^{7}$. The regions specifically involved in semantic processing is a current topic of debate, with multiple competing models ${ }^{28,38-40}$, and we defined a list of semantic ROls by combining across these models. Using a family of plausible candidate ROIs for this comparison dilutes the importance of including or excluding any particular ROI. Taken together, the results firmly place STSU and STSL at different levels of the auditory cortical hierarchy. 
Multiple lines of evidence support a pathway linking auditory cortical and limbic structures ${ }^{69-72}$ that subserves auditory memory ${ }^{14,17,18}$ and affective sound processing ${ }^{73}$. The data presented here contribute to our understanding of this pathway. Clustering analysis identified a set of ROIs (PP, InsP, STGA, InsA, TP) positioned between auditory and limbic cortex (Fig. 4). PP is anatomically close and connected to auditory cortex ${ }^{74}$, yet it is unique in auditory-responsive cortex for its syntactic-level language processing ${ }^{3}$ and its preferential activation by music, which has a strong affective component ${ }^{4}$. The recently reported connectivity between Hippocampus and $\mathrm{PP}^{75}$ is consistent with PP's role as an intermediate node in this stream. Similarly, InsP, despite its anatomical proximity and overlapping response properties with HGPM, is likely involved in the transformation of auditory information in auditory cortex to affective

372 representations in $\operatorname{Ins} A^{5}$.

373 The ATL structures STGA and TP are involved in semantic processing ${ }^{3,28}$ and auditory memory ${ }^{76}$,

374 in particular the representation and retrieval of memories for people, social language, and

375 behaviors ('social knowledge') ${ }^{77}$. Tight clustering of TP with limbic ROIs in embedding space is TP shares key features of laminar cytoarchitecture and strong connectivity ${ }^{75}$. We suggest that the organization depicted in Figures 3 and 4, combined with evidence for bidirectional information sharing between auditory cortex and limbic areas, merits the identification of a third auditory processing stream alongside the dorsal and ventral streams ${ }^{8,80}$. This 'limbic stream' would underlie auditory contributions to affective and episodic memory processing.

\section{Ventral and dorsal streams linking auditory and frontal cortex}

Current models of speech and language processing posit the existence of ventral and dorsal processing streams linking non-core auditory cortex with PMC and inferior frontal gyrus via Despite substantial experimental evidence supporting these models, there is a lack of consensus on the specific functions subserved by the two streams. For example, the dorsal stream has been envisioned to subserve spatial processing $\left(\right.$ "where" $\left.{ }^{8}\right)$, sensorimotor integration ("how" ${ }^{\prime 9}$ ), and syntactic processing ${ }^{10}$. There is a parallel debate about the specific cortical regions comprising the two streams.

As broadly predicted by these models, temporal and parietal ROls segregated in embedding space in the analysis presented here (Fig. 3b, 4). We observed a cluster that included STSL, middle and inferior temporal gyrus ROIs, in conformity with the ventral auditory stream proposed by Hickok and Poeppel ${ }^{9}$ and Friederici ${ }^{10}$. By contrast, the cluster that included SMG, $A G P$, and AGA aligned with the dorsal processing stream as proposed by Rauschecker and

$397 \mathrm{Scott}^{8}$. Association of FG and MOG with the ventral and dorsal clusters, respectively, likely represents the sharing of information across sensory modalities. 
A previous $\mathrm{fMRI}$-based DME study found that primary sensory and default mode ROIs segregated along the first dimension in embedding space ${ }^{32}$. Coverage of mesial cortex in our dataset was limited, precluding a direct comparison. However, the striking separation between auditory and prefrontal cortex in embedding space shown here, and its robustness to the choice of the parameter $t$, indicate that the current results align well with the previous report. This separation places auditory and frontal regions at opposite ends of the auditory processing hierarchy, linked by ventral and dorsal processing streams ${ }^{8-10}$.

\section{Network hubs}

Hubs in brain networks play a critical role in integrating distributed neural activity ${ }^{46}$. In the present analysis, global hubs were characterized by their central location within embedding space and high mean connectivity (Fig. 5). These hubs included STGA and MTGA, both components of the ATL. Previous reports indicate that ATL serves as a transmodal hub, transforming sensory domain-specific to domain-general representations ${ }^{28,81,82}$ and playing a central role in semantic processing and social memory ${ }^{28,77,83}$. MTGM also appears as a global hub, even though it is not formally part of the ATL. Interestingly, patients with semantic dementia have ATL degeneration ${ }^{84,85}$, but the damage is often more widespread and can include $\mathrm{MTGM}^{86}$.

Several other ROls also exhibited hub-like properties. These included ITGA (part of ATL), the well-established global hub PCC/preCun ${ }^{32}$, and STSL. The latter observation is consistent with the diverse and multimodal functionality attributed to the STS previously ${ }^{63,87}$.

Unlike other ATL structures, TP does not appear as a global hub in Figure 5b. The close association of TP with limbic structures in embedding space suggests that TP mediates interactions between multimodal integration centers in the ATL and structures subserving memory functions. More broadly, the heterogeneity of ATL ROIs in terms of their global hublike connectivity profiles conforms to the observation that the terminal fields of white matter tracts converging in the ATL only partially overlap ${ }^{28,88,89}$.

\section{Hemispheric lateralization}

Although speech and language networks are classically described as highly lateralized, imaging studies have demonstrated widespread bilateral activation during speech and language tasks ${ }^{90-}$ 92. We found no evidence for hemispheric differences in cortical functional organization based on analysis of all sampled brain regions, nor when comparison was restricted to speech and language ROIs (Fig. 6). These results are consistent with a recent fMRI study ${ }^{26}$ showing similar RS connectivity patterns between left and right temporal lobe (but see ${ }^{6}$ ). 
A recent study that applied DME to RS-fMRI data presented evidence for hemispheric asymmetry in semantic networks, which correlated with semantic task performance ${ }^{93}$. The large dataset in that study and the availability of data from both hemispheres in every participant likely facilitated detection of those differences. Another possible reason for this divergence is that we investigated hemispheric differences based on all relevant dimensions of embedding space, whereas the previous study relied only on position along the first dimension. In addition, hemispheric asymmetry in network connectivity increases along the cortical hierarchy ${ }^{94}$. Thus, ROIs involved in heteromodal semantic processing would likely show greater hemispheric differences compared to speech and language ROIs, which include lower order sensory regions. The present study suggests that neither anatomical nor task-specific functional hemispheric asymmetries necessarily translate into asymmetric network configurations during resting state. This does not exclude the possibility of asymmetries emerging during auditory tasks, for example reflecting hemispheric biases in spectral and temporal processing ${ }^{9,12}$.

\section{Caveats \& limitations}

A key concern regarding all human iEEG studies is that participants may not be representative of a healthy population. In the present study, results were consistent across participants despite differences in seizure disorder histories, medications, and seizure foci, and aligned with results obtained previously in healthy participants ${ }^{32}$. Another caveat is that our dataset, however extensive, did not sample the entire brain, and it was not possible to infer connectivity with unsampled regions. To address this, we applied DME analysis to fMRI data to establish that the organization of ROIs in embedding space was robust to the exclusion of unsampled ROIs. Although there was a greater effect of sparse, non-uniform sampling within an ROI, there was still considerable similarity in functional organization to embeddings derived from averages across the entire ROI.

While subcortical structures (e.g., thalamus) that link sensory and higher order networks ${ }^{95}$ were not sampled, the functional organization presented here was likely influenced indirectly by thalamo-cortical pathways ${ }^{61,96}$. Previous fMRI studies of RS networks focused exclusively on cortical ROls and did not consider the role of the thalamus and other subcortical structures. Despite this limitation, these studies have yielded valuable insights into the functional organization of the human cortical networks ${ }^{97,98}$.

\section{Concluding remarks and future directions}

This study extends the DME approach to characterize functional relationships between cortical regions investigated using iEEG recordings. These data help resolve several outstanding issues regarding the functional organization of human auditory cortical networks and stress the importance of a limbic pathway complementary to the dorsal and ventral streams. These 
471 results lay the foundation for future work investigating network organization during active

472 speech and language processing. While the current work focused on auditory cortical networks,

473 this approach can be readily generalized to advance our understanding of changes in brain

474 organization during sleep and anesthesia, disorders of consciousness, as well as reorganization

475 of cortical functional geometry secondary to lesions. 


\section{Online Methods}

477

478

479

480

481

482

483

484

485

486

487

488

489

490

491

492

493

494

495

496

497

498

499

500

501

502

503

504

505

506

507

508

509

510

511

512

\section{Participants}

The study was carried out in 46 neurosurgical patients (20 females) diagnosed with medically refractory epilepsy. The patients were undergoing chronic invasive electrophysiological monitoring to identify seizure foci prior to resection surgery (Supplementary Table 1). Research protocols were approved by the University of lowa Institutional Review Board and the National Institutes of Health, and written informed consent was obtained from all participants. Research participation did not interfere with acquisition of clinically necessary data, and participants could rescind consent for research without interrupting their clinical management.

All participants except two were native English speakers. The participants were predominantly right-handed (39 out of 46); six participants were left-handed, and one had bilateral handedness. The majority of participants (32 out of 46 ) were left language-dominant, as determined by Wada test. Two participants were right hemisphere-dominant, and one had bilateral language dominance. The remaining 11 participants were not evaluated for language dominance; 9 of them were right-handed and thus were assumed left language-dominant for the purposes of the analysis of lateralization (see below). The remaining two participants who did not undergo Wada test and who were left-handed were not included in that analysis.

All participants underwent audiological and neuropsychological assessment prior to electrode implantation, and none had auditory or cognitive deficits that would impact the results of this study. The participants were tapered off their antiepileptic drugs during chronic monitoring when RS data were collected.

\section{Experimental procedures}

Pre-implantation neuroimaging. All participants underwent whole-brain high-resolution T1weighted structural MRI scans before electrode implantation. In a subset of ten participants (Supplementary Table 2), RS-fMRI data were used for estimates of functional connectivity. The scanner was a 3T GE Discovery MR750W with a 32-channel head coil. The pre-electrode implantation anatomical T1 scan (3D FSPGR BRAVO sequence) was obtained with the following parameters: $\mathrm{FOV}=25.6 \mathrm{~cm}$, flip angle $=12 \mathrm{deg}$., $\mathrm{TR}=8.50 \mathrm{~ms}, \mathrm{TE}=3.29 \mathrm{~ms}$, inversion time $=$ $450 \mathrm{~ms}$, voxel size $=1.0 \times 1.0 \times 0.8 \mathrm{~mm}$. For RS-fMRI, 5 blocks of 5 -minute gradient-echo EPI runs (650 volumes) were collected with the following parameters: $F O V=22.0 \mathrm{~cm}, \mathrm{TR}=2260$ $\mathrm{ms}, \mathrm{TE}=30 \mathrm{~ms}$, flip angle $=80$ deg., voxel size $=3.45 \times 3.45 \times 4.0 \mathrm{~mm}$. In some cases, fewer RS acquisition sequences were used in the final analysis due to movement artifact or because the full scanning session was not completed. For each participant, RS-fMRI runs were acquired in the same session but non-contiguously (dispersed within an imaging session to avoid habituation). Participants were asked to keep their eyes open, and a fixation cross was presented through a projector. 
513 iEEG recordings. iEEG recordings were obtained using either subdural and depth electrodes, or

514 depth electrodes alone, based on clinical indications. Electrode arrays were manufactured by

515 Ad-Tech Medical (Racine, WI). Subdural arrays, implanted in 36 participants out of 46, consisted

516 of platinum-iridium discs (2.3 $\mathrm{mm}$ diameter, 5-10 $\mathrm{mm}$ inter-electrode distance), embedded in a

517 silicon membrane. Stereotactically implanted depth arrays included between 4 and 12

518 cylindrical contacts along the electrode shaft, with 5-10 mm inter-electrode distance. A

519 subgaleal electrode, placed over the cranial vertex near midline, was used as a reference in all

520 participants. All electrodes were placed solely on the basis of clinical requirements, as

521 determined by the team of epileptologists and neurosurgeons ${ }^{99}$.

522 No-task RS data were recorded in the dedicated, electrically shielded suite in The University of

523 lowa Clinical Research Unit while the participants lay in the hospital bed. RS data were collected

$5246.4+$ +/- 3.5 days (mean \pm standard deviation; range 1.5 - 20.9) after electrode implantation

525 surgery. In the first 15 participants (L275 through L362), data were recorded using a TDT RZ2

526 real-time processor (Tucker-Davis Technologies, Alachua, FL). In the remaining 31 participants

527 (R369 through L585), data acquisition was performed using a Neuralynx Atlas System

528 (Neuralynx Inc., Bozeman, MT). Recorded data were amplified, filtered $(0.1-500 \mathrm{~Hz}$ bandpass, 5

$529 \mathrm{~dB} /$ octave rolloff for TDT-recorded data; $0.7-800 \mathrm{~Hz}$ bandpass, $12 \mathrm{~dB} /$ octave rolloff for

530 Neuralynx-recorded data) and digitized at a sampling rate of $2034.5 \mathrm{~Hz}$ (TDT) or $2000 \mathrm{~Hz}$

531 (Neuralynx). The durations of recordings were 13 +/- $11 \mathrm{~min}$. In all but two participants,

532 recording durations were between 10 and 22 min.; in one participant duration was 6 min., and

533 in one participant the duration was $81 \mathrm{~min}$.

534

535

536

537

538

539

540

541

542

543

544

545

546

547

548

549

550

\section{Data analysis}

Anatomical reconstruction and ROI parcellation. Localization of recording sites and their assignment to ROIs relied on post-implantation T1-weighted anatomical MRI and postimplantation computed tomography (CT). All images were initially aligned with pre-operative T1 scans using linear coregistration implemented in FSL (FLIRT) ${ }^{100}$. Electrodes were identified in the post-implantation MRI as magnetic susceptibility artifacts and in the CT as metallic hyperdensities. Electrode locations were further refined within the space of the pre-operative MRI using three-dimensional non-linear thin-plate spline warping ${ }^{101}$, which corrected for postoperative brain shift and distortion. The warping was constrained with 50-100 control points, manually selected throughout the brain, which were visually aligned to landmarks in the preand post-implantation MRI.

To pool data across participants, the dimensionality of connectivity matrices was reduced by assigning electrodes to one of 58 ROIs organized into 6 ROI groups (see Fig. 1; Supplementary Table 2,3 ) based upon anatomical reconstructions of electrode locations in each participant. For subdural arrays, ROI assignment was informed by automated parcellation of cortical gyri $^{102,103}$ as implemented in the FreeSurfer software package. For depth arrays, it was informed 
by MRI sections along sagittal, coronal, and axial planes. For recording sites in Heschl's gyrus, delineation of the border between core auditory cortex adjacent non-core areas (HGPM and HGAL, respectively) was performed in each participant using physiological criteria ${ }^{104,105}$. Specifically, recording sites were assigned to HGPM if they exhibited phase-locked (frequencyfollowing) responses to $100 \mathrm{~Hz}$ click trains and if the averaged evoked potentials to these stimuli featured short-latency (<20 ms) peaks. Such response features are characteristic for HGPM and are not present within $\mathrm{HGAL}^{104}$. Additionally, correlation coefficients between average evoked potential waveforms recorded from adjacent sites were examined to identify discontinuities in response profiles along Heschl's gyrus that could be interpreted as reflecting a transition from HGPM to HGAL. Superior temporal gyrus was subdivided into posterior and middle non-core auditory cortex ROIs (STGP and STGM), and auditory-related anterior ROI (STGA) using the transverse temporal sulcus and ascending ramus of the Sylvian fissure as macroanatomical boundaries. The insula was subdivided into posterior and anterior ROls, with the former considered within the auditory-related ROI group ${ }^{5}$. Middle and inferior temporal gyrus were each divided into posterior, middle, and anterior ROIs by diving the gyrus into three approximately equal-length thirds. Angular gyrus was divided into posterior and anterior ROIs using the angular sulcus as a macroanatomical boundary. Anterior cingulate cortex was identified by automatic parcellation in FreeSurfer and was considered as part of the prefrontal ROI group, separately from the rest of the cingulate gyrus. Postcentral and precentral gyri were each divided into ventral and dorsal portions using the $y_{\mathrm{MNI}}$ coordinate (see below) of $40 \mathrm{~mm}$ as a boundary. Recording sites identified as seizure foci or characterized by excessive noise, and depth electrode contacts localized to the white matter or outside brain, were excluded from analyses and are not listed in Supplementary Table 2.

Preprocessing of fMRI data. Standard preprocessing was applied to the RS-fMRI data acquired in the pre-implantation scan using FSL's FEAT pipeline, including spatial alignment and nuisance regression. White matter, cerebrospinal fluid and global ROls were created using deep white matter, lateral ventricles and a whole brain mask, respectively. Regression was performed using the time series of these three nuisance ROls as well as 6 motion parameters ( 3 rotations and 3 translations) and their derivatives, detrended with second order polynomials. Temporal bandpass filtering was $0.008-0.08 \mathrm{~Hz}$. Spatial smoothing was applied with a Gaussian kernel (6 $\mathrm{mm}$ full-width at half maximum). The first two images from each run were discarded. Frame censoring was applied when the Euclidean norm of derivatives of motion parameters exceeded $0.5 \mathrm{~mm}^{106}$. All runs were processed in native EPI space, then the residual data were transformed to MNI152 and concatenated.

Preprocessing of iEEG data. Analysis of iEEG data was performed using custom software written in MATLAB Version 2020a programming environment (MathWorks, Natick, MA, USA). After initial rejection of recording sites identified as seizure foci, several automated steps were taken to exclude recording channels and time intervals contaminated by noise. First, channels were excluded if average power in any frequency band (broadband, delta, theta, alpha, beta, gamma, or high gamma; see below) exceeded 3.5 standard deviations of the average power across all 
channels for that participant. Next, transient artifacts were detected by identifying voltage deflections exceeding 10 standard deviations on a given channel. A time window was identified extending before and after the detected artifact until the voltage returned to the zero-mean baseline plus an additional $100 \mathrm{~ms}$ buffer before and after. High-frequency artifacts were also removed by masking segments of data with high gamma power exceeding 5 standard deviations of the mean across all segments. Only time bins free of these artifact masks were considered in subsequent analyses. Artifact rejection was applied across all channels simultaneously so that all connectivity measures were derived from the same time windows. Occasionally, particular channels survived the initial average power criteria yet had frequent artifacts that led to loss of data across all the other channels. There is a tradeoff in rejecting artifacts (losing time across all channels) and rejecting channels (losing all data for that channel). If artifacts occur on many channels, there is little benefit to excluding any one channel. However, if frequent artifacts occur on one or simultaneously on up to a few channels, omitting these can save more data from other channels than those channels contribute at all other times. We chose to optimize the total data retained, channels $\times$ time windows, and omitted some channels when necessary. To remove shared signals unlikely to derive from brain activity, data from retained channels were high-pass filtered above $200 \mathrm{~Hz}$, and a spatial filter was derived from the singular value decomposition omitting the first singular vector. This spatial filter was then applied to the broadband signal to remove this common signal.

Connectivity analysis. For RS-fMRI data, BOLD signals were averaged across voxel groupings and functional connectivity was calculated as Pearson correlation coefficients. Voxel groupings were either based on the Schaefer-Yeo 400 parcellation scheme ${ }^{53}$ in MNI-152 space, or were based on iEEG electrode location in participant space (see Fig. 1). For the latter, fMRI voxels were chosen to represent comparable regions of the brain recorded by iEEG electrodes. For each electrode, the anatomical coordinates of the recording site were mapped to the closest valid MRI voxel, $E$, and a sphere of 25 voxels $\left(25 \mathrm{~mm}^{3}\right)$ centered on $E$ used as the corresponding recording site. This process was repeated for all $N$ electrodes in the same ROI, and a single time series computed as the average of the fMRI BOLD signal in these $N \times 25$ voxels. These averages were used to compute an ROI-by-ROI connectivity matrix for RS-fMRI data. For comparisons between iEEG and fMRI embeddings, voxels were processed in participant space and ROI labels from the parcellation scheme illustrated in Figure 1 and Supplementary Table 2 were applied to the $\mathrm{fMRI}$ data. For comparisons between $\mathrm{fMRI}$ embeddings derived from all cortical ROls versus $\mathrm{fMRI}$ embeddings derived from just ROIs sampled in the iEEG experiments, electrode locations were transformed from participant space to MNI-152 space, then assigned to ROls within the Schaefer-Yeo 400 scheme.

For iEEG data, two measures of functional (non-directed) connectivity were used: the orthogonalized band power envelope correlation ${ }^{29}$ and the debiased weighted phase lag index $\left(\right.$ wPLI; ${ }^{52}$ ), a measure of phase synchronization. Both measures avoid artifacts due to volume conduction by discounting connectivity near zero phase lag. For both measures, data were 
divided into 60-second segments, pairwise connectivity estimated in each segment, and then connectivity estimates averaged across all segments for that participant.

Envelope correlations were estimated for each data segment and every recording site as in ${ }^{29}$, except time-frequency decomposition was performed using the demodulated band transform $^{107}$, rather than wavelets. For each frequency band (theta: 4-8 Hz, alpha: 8-13 Hz, beta: $13-30 \mathrm{~Hz}$, gamma: $30-70 \mathrm{~Hz}$; high gamma: 70-120 Hz), the power at each time bin was calculated as the average (across frequencies) log of the squared amplitude. For each pair of signals $X$ and $Y$, one was orthogonalized to the other by taking the magnitude of the imaginary component of the product of one signal with the normalized complex conjugate of the other:

$$
Y_{\text {orth }}=\left|\operatorname{Im}\left\{Y \times X^{*} /|X|\right\}\right|
$$

Both signals were band-pass filtered $(0.2-1 \mathrm{~Hz})$, and the Pearson correlation calculated between signals. The process was repeated by orthogonalizing in the other direction and the overall envelope correlation for a pair of recording sites was the average of the two Pearson correlations.

wPLI was estimated for each data segment and every recording site pair from the sign of the imaginary part of the cross-spectrum at each frequency and averaged across frequencies within each band of interest (theta: 4-8 Hz, alpha: 8-13 Hz, beta: 13-30 Hz). The cross spectrum was calculated from the demodulated band transform as described previously ${ }^{108}$.

Prior to diffusion map embedding, connectivity matrices were thresholded by saving at least the top third (rounded up) connections for every row, as well as their corresponding columns (to preserve symmetry). We also included any connections making up the minimum spanning tree of the graph represented by the elementwise reciprocal of the connectivity matrix to ensure the graph is connected.

ROI-based connectivity analysis. Connectivity between ROIs was computed as the average envelope correlation or WPLI value between all pairs of recording sites in the two ROIs. For analyses in which connectivity was summarized across participants (Fig. 3-8), we used only a subset of ROls such that every possible pair of included ROIs was represented in at least two participants (Supplementary Table 2). This list of ROIs was obtained by iteratively removing ROIs with the worst cross-coverage with other ROIs until every ROI remaining had sufficient coverage with all remaining ROls.

Diffusion map embedding. See the Appendix for details about DME. In brief, the connectivity matrix $\mathbf{K}=[k(i, j)]$ is normalized by degree to yield a transition probability matrix $\mathbf{P}$. DME consists of performing an eigendecomposition of $\mathbf{P}$, with eigenvalues scaled according to the free parameter $t$, which sets the spatial scale of the analysis. If the recording sites are conceptualized as nodes on a graph with edges defined by $\mathbf{K}$, then $\mathbf{P}$ can be understood as the transition probability matrix for a 'random walk' or a 'diffusion' on the graph (see Appendix; ${ }^{30,31}$ ). The parameter $t$ is the number of time steps in that random walk; larger values 
of $t$ correspond to exploring the structure of the data at larger spatial scales. In the analyses presented here, $k(i, j)$ is either the orthogonalized power envelope correlations, or the debiased WPLI. We note that in recent applications of DME to fMRI functional connectivity data, $\mathbf{K}$ was first transformed by applying cosine similarity ${ }^{32}$. However, this additional step has not been universally implemented (e.g., ${ }^{109}$ ), nor is it required within the mathematical framework of $\mathrm{DME}^{30,31}$. In the case of the dataset presented here, applying cosine similarity to the data served to smear the data in embedding space, increasing variance, and decreasing separation between ROls, but otherwise produced qualitatively similar results.

In two sets of analyses presented here, pairs of embeddings were compared to each other: in the analysis of lateralization of speech and language networks, and in the comparison between iEEG and $\mathrm{fMRI}$ data. To do that, we used a change of basis operator to map embeddings into a common embedding space using the method described in Coifman et al $2014^{31}$.

Dimensionality reduction via low rank approximations to $\mathbf{P}$. Diffusion map embedding offers an opportunity to reduce the dimensionality of the underlying data by considering only those dimensions that contribute importantly to the structure of the data, as manifested in the structure of the transition probability matrix $\mathbf{P}$. We used the eigenvalue spectrum of $\mathbf{P}$ to determine its ideal low rank approximation, balancing dimensionality reduction and information loss. Because $\mathbf{P}$ is real and symmetric, the magnitude of the eigenvalues is identical to the singular values of $\mathbf{P}$. The singular values tell us about the fidelity of low rank approximations to $\mathbf{P}$. Specifically, if $\mathbf{P}$ has a set of singular values $\sigma_{1} \geq \sigma_{1} \geq \ldots \geq \sigma_{n}$, then for any integer $k \geq 1$,

$$
\min _{\widehat{\mathbf{P}_{k}}}\left\|\mathbf{P}-\widetilde{\mathbf{P}_{k}}\right\|_{2}=\sigma_{k+1},
$$

where $\widetilde{\mathbf{P}_{k}}$ is the rank-k approximation to $\mathbf{P}$. Thus, the magnitude of the eigenvalues corresponds to the fidelity of the lower dimensional approximation, and the difference in the magnitude of successive eigenvalues represents the improvement in that approximation as the dimensionality increases. The spectrum of $\mathbf{P}$ invariably has an inflection point ("elbow"), separating two sets of eigenvalues $\lambda_{i}$ : those whose magnitude decreases more quickly with increasing $i$, and those beyond the inflection point whose magnitude decreases more slowly with increasing $i$. The inflection point thus delineates the number of dimensions that are most important for approximating $\mathbf{P}$. The inflection point $k_{\text {infl }}$ was identified algorithmically ${ }^{110}$, and the number of dimensions retained set equal to $k_{\text {infl }}-1$.

Comparing distances in embedding space. The relative distance between points in embedding space provides insight into the underlying functional geometry. In several analyses presented here, two embeddings of identical sets of ROls were compared as ROI distances within the two embeddings. After mapping to a common space and reducing dimensionality as described above, the two embeddings $A$ and $B$ were used to create the pairwise distance matrices $A$ ' and $B$ '. The Pearson correlation coefficient $r$ was then computed between the upper triangles (excluding the diagonal) of the corresponding elements in the distance matrices. 
Signal to noise (SNR) characteristics. To measure the robustness of the embedding analysis to variability over time, an SNR was computed as follows. For each participant, a channel $\times$ channel P matrix was calculated for each $60 \mathrm{~s}$ segment of data. For each segment, DME analysis was applied and a channel $\times$ channel distance matrix calculated. These distance matrices were averaged across segments. The 'signal' of interest was defined as the variability (standard deviation) of this averaged distance matrix (ignoring the diagonals). The 'noise' was defined as the variability across time, estimated for each element of the distance matrix as the standard deviation across segments, then averaged across the elements of the matrix. The SNR for functional connectivity itself was computed in an analogous manner, using the original channel $x$ channel connectivity matrix rather than the matrix of embedding distances.

Estimating precision in position and distances in embedding space. To obtain error estimates for both ROI locations in embedding space and embedding distance between ROls, average ROI $x$ ROI adjacency matrices were calculated. This was done by drawing each edge from an averaged bootstrap sample across participants, obtaining 10,000 such adjacency matrices, and performing diffusion map embedding for each. For locations in embedding space, these embeddings were then mapped via the change of basis procedure described above to the original group average embedding space. For each ROI, the mapped bootstrap iterations produced a cloud of locations in embedding space which were summarized by the standard deviation in each dimension. For embedding distances, no change of basis was necessary because distances were preserved across bases.

To compare the positions of STSL versus STSU relative to canonical auditory cortical ROIs (HGPM, HGAL, PT, PP, STGP, and STGM) or ROls involved in semantic processing (STGA, MTGA, MTGP, ITGA, ITGP, TP, AGA, AGP, SMG, IFGop, IFGtr, IFGor ${ }^{28,38-40}$ ), we calculated the average pairwise distance from STSL or STSU to each such ROI. The difference between these averages was compared to a null distribution obtained by Monte Carlo sampling of the equivalent statistic obtained by randomly exchanging STSL/STSU labels by participant. The specific comparisons performed were chosen a priori to constrain the number of possible hypotheses to test; pairwise comparisons of all possible ROI pairs (let alone comparisons of all higher-order groupings) would not have had sufficient statistical power under appropriate corrections for multiple comparisons. Though different choices could have been made for inclusion in the "semantic processing" category, exchanging one or two of these ROls would not strongly influence the average distance in a group of twelve ROIs.

Hierarchical clustering. Agglomerative hierarchical clustering was done using the linkage function in MATLAB, with Euclidean distance as the distance metric and Ward's linkage (minimum variance algorithm) as the linkage method. The ordering of ROls along the horizontal axis in the dendrogram was determined using the optimalleaforder function in MATLAB, with the optimization criterion set to 'group'.

Comparing language dominant/non-dominant hemispheres. To test for differences in embedding location between language dominant and non-dominant hemispheres, two 
742 measures were considered: different location of individual ROIs in embedding space, and

743 different pairwise distances between ROIs in embedding space. To calculate differences in

744 location of individual ROIs, dominant/non-dominant average embeddings were mapped to a

745 common space (from an embedding using the average across all participants regardless of

746 language dominance) using the change of basis operator. The language-dominant location

747 difference for a specific ROI was calculated as the Euclidean distance between the two locations

748 of each ROI in this common space. For pairwise distances, the change of basis is irrelevant, so

749 pairwise Euclidean distances were calculated in embedding space for each hemisphere and

750 then subtracted to obtain a difference matrix. To determine whether the differences in location

751 or pairwise distances were larger than expected by chance, random permutations of the

752 dominant/non-dominant labels were used to generate empirical null distributions. Since this

753 approach produces a $p$-value for every pair of connections, $p$-values were adjusted using false

754 discovery rate (FDR) to account for multiple comparisons.

755 Analyses of fMRI connectivity in embedding space. Two sets of analyses were performed using

$756 \mathrm{fMRI}$ data. First, iEEG and fMRI data were compared in embedding space. In this analysis,

757 connectivity based on RS-fMRI data from voxels located at electrode recording sites was

758 compare with the corresponding connectivity matrix derived from iEEG data. The embedding

759 analysis was applied to the two connectivity matrices, all pairwise inter-ROI distances

760 computed, and $\mathrm{IEEG}$ and $\mathrm{fMRI}$ data compared using the correlation of the pairwise ROI

761 distances. The second analysis was to compare embeddings derived from all ROls in the RS-

$762 \mathrm{fMRI}$ scans to those derived from just ROIs sampled with iEEG electrodes. Here, ROI $\times$ ROI

763 connectivity matrices were computed for all ROls, then embeddings created from the full

764 matrices or from matrices containing just rows and columns corresponding to the ROls sampled

765 with iEEG. 


\section{Appendix: Diffusion Map Embedding}

767

768

769

770

771

772

773

774

775

776

777

778

779

780

781

782

783

784

785

786

787

788

789

790

791

792

793

794

795

796

797

798
In the framework of DME, we consider a space $X$ that is the set of $N$ recording sites. We compute the similarity between those sites based on the time varying signals recorded at each site, defining similarity as the functional connectivity measure $k\left(x_{i}, x_{j}\right)$.

Define the matrix $\mathbf{K}$ whose $i, j^{\text {th }}$ element is $k\left(x_{i}, x_{j}\right) . k\left(x_{i}, x_{j}\right)$ is required to be symmetric, i.e., $k\left(x_{i}, x_{j}\right)$ $=k\left(x_{j}, x_{i}\right)$, and positivity preserving, i.e. $k\left(x_{i}, x_{j}\right)>0$ for all $[i, j]$, to allow for spectral analysis of a normalized version of $\mathbf{K}$.

From $X$ and $\mathbf{K}$ we can construct a weighted graph $\Gamma$ in which the vertices are the nodes and the edge weights are $k\left(x_{i}, x_{j}\right)$. We take random walks on the graph at time steps $t=1,2, \ldots$, jumping from node $x_{i}$ to node $x_{j}$ at each time step, with the (stochastic) decision as to which node should be visited next depending on $k\left(x_{i}, x_{j}\right)$.

Define

$$
p\left(x_{i}, x_{j}\right)=k\left(x_{i}, x_{j}\right) / d\left(x_{i}\right)
$$

where

$$
d\left(x_{i}\right)=\Sigma_{j}\left[k\left(x_{i}, x_{j}\right)\right]
$$

is the degree of node $x_{i}$. Normalizing $k\left(x_{i}, x_{j}\right)$ in this way allows us to interpret it as the probability $p\left(x_{i}, x_{j}\right)$ that we'll jump from vertex $x_{i}$ to vertex $x_{j}$ in a single time step of our random walk.

If we consider a single time step, we only capture the structure in $X$ on a very local scale, since we can only jump between vertices that are directly connected. As we run the random walk forward in time, we begin to explore more of our neighborhood, and we begin to explore other neighborhoods as well. Two vertices $x_{i}$ and $x_{j}$ that have similar connectivity to the rest of the network have a high probability of being connected during these longer walks because they themselves are connected to similar groups of vertices, and so there are many possible paths between $x_{i}$ and $x_{j}$.

The diffusion operator (matrix) $\mathbf{P}=\left[p\left(x_{i}, x_{j}\right)\right]$ describes how signals diffuse from node to node in the graph. If $\mathbf{v}$ is a $N \times 1$ vector (i.e., a value assigned to each vertex, for example representing an input to each node), then $\mathbf{P}$ describes what will happen to that input as time goes on.

$$
\mathbf{P v}=\left[p\left(x_{1}, x_{1}\right) \mathbf{v}\left[x_{1}\right]+p\left(x_{1}, x_{2}\right) \mathbf{v}\left[x_{2}\right]+\ldots ; p\left(x_{2}, x_{1}\right) \mathbf{v}\left[x_{1}\right]+p\left(x_{2}, x_{2}\right) \mathbf{v}\left[x_{2}\right]+\ldots ; \ldots\right]^{\top}
$$

If, for example, all the nodes were insular, with $p\left(x_{i}, x_{i}\right)=1$ for all $i$, and otherwise $p\left(x_{i}, x_{j}\right)=0, \mathbf{P v}=$ $\mathbf{v}$, i.e., no diffusion occurs. If the probabilities are more distributed, Pv would reveal how much signals diffuse out from each node given the starting condition of $\mathbf{v}$. Importantly, $\mathbf{P}^{\mathbf{k}} \mathbf{v}$ would reveal what that distribution looks like after $k$ time steps. 
The eigenvector expansion of $\mathbf{P}$ based on its eigenvectors $\psi_{j}$ and eigenvalues $, \lambda_{j}, j=1 \ldots N$, is a natural method for uncovering structure in $\mathbf{P}$ because each eigenvector of $\mathbf{P}$ is a dimension along which relevant organizational features emerge. That is, clusters of related points (communities) tend to be distinct and ordered along these dimensions. In fact, we could preserve a lot of information about $\mathbf{P}$ by keeping just a subset of $M$ of these vectors and discarding the rest. The information we want to preserve in the context of diffusion map embedding is the functional distance between the data at two nodes given $t$ time steps to meander through the graph. We can define the diffusion map

$$
\Psi^{(t)}\left(x_{i}\right)=\left[\lambda_{1}{ }^{t} \psi_{1}\left(x_{i}\right), \lambda_{1}{ }^{t} \psi_{1}\left(x_{i}\right), \ldots, \lambda_{M}{ }^{t} \psi_{M}\left(x_{i}\right)\right]^{\top},
$$

which maps each point $x$ in $X$ to a point in an embedding space of dimension $M \leq N$. In this space, the diffusion distance $D$, which is the Euclidean distance between points, is the difference in the probability distributions linking $x_{i}$ to the rest of the network and $x_{j}$ to the rest of the network:

$$
D^{(t)}\left(x_{i}, x_{j}\right)^{2}=\left\|\mid \Psi^{(t)}\left(x_{i}\right)-\Psi^{(t)}\left(x_{j}\right)\right\|_{12}=\left\|p^{(t)}\left(x_{i},:\right)-p^{(t)}\left(x_{j},:\right)\right\|^{2} / 2 .
$$

We return now to the parameter $t$, which corresponds to the time scale of the diffusion process (i.e., the number of steps in the random walk on the graph). As $t$ progresses, the coordinates of the data in embedding space are scaled according to $\lambda_{i}{ }^{t}$, where $\lambda_{i}$ is the eigenvalue of the $i^{\text {th }}$ dimension being scaled. Thus, the value of $t$ sets the spatial scale of the analysis, with higher values de-emphasizing smaller eigenvalues. Because $\left|\lambda_{i}\right|<1 \forall i$, at higher values of $t$ each dimension will be scaled down ('collapse'), with the dimension corresponding to $\max \left(\left|\lambda_{i}\right|\right)$ (i.e., $\lambda_{1}$ ) scaled the least.

To compare embeddings across groups of participants, or modalities of measurements, it is necessary to map embeddings to a common space. To do so, consider two sets of data $\alpha$ and $\beta$, and the data spaces $X_{\alpha}$ and $X_{\beta}$. The problem is that $X_{\alpha}$ and $X_{\beta}$ are different spaces with different kernels $k_{\alpha}$ and $k_{\beta}$. This means that the eigenvectors for $\mathbf{P}_{\alpha}$ and $\mathbf{P}_{\beta}$ will be different, and data projected into a space defined by some subset of the eigenvectors cannot be compared directly. The solution is to apply a change of basis operator to one set of the eigenvectors to get the data into the same embedding space ${ }^{31}$ :

$$
\mathrm{D}^{(t)}\left(x_{i \mid \alpha}, x_{i \mid \beta}\right)=\left\|\Psi_{\alpha}^{(t)}(x)-\mathrm{O}_{\beta \rightarrow \alpha} \Psi_{\beta}^{(t)}(x)\right\|_{12} .
$$

Where the change of basis operator $\mathrm{O}_{\beta \rightarrow \alpha}$ is defined as

$$
\mathrm{O}_{\beta \rightarrow \alpha} \mathbf{v}=\Sigma_{j}\left[\mathbf{v}(j)\left\langle\psi_{\alpha}^{(i)},_{\beta}^{(j)}\right]_{i>=1},\right.
$$

Where $\langle\mathbf{a}, \mathbf{b}\rangle$ is the inner product of $\mathbf{a}$ and $\mathbf{b}$. 
1. Scott, S.K. The neurobiology of speech perception and production--can functional imaging tell us anything we did not already know? J Commun Disord 45, 419-425 (2012).

2. Woods, D.L. \& Alain, C. Functional imaging of human auditory cortex. Curr Opin Otolaryngol Head Neck Surg 17, 407-411 (2009).

3. Friederici, A.D., Meyer, M. \& von Cramon, D.Y. Auditory language comprehension: an event-related fMRI study on the processing of syntactic and lexical information. Brain Lang 75, 289-300 (2000).

4. Angulo-Perkins, A., et al. Music listening engages specific cortical regions within the temporal lobes: differences between musicians and non-musicians. Cortex 59, 126-137 (2014).

5. Zhang, Y., et al. The Roles of Subdivisions of Human Insula in Emotion Perception and Auditory Processing. Cereb Cortex 29, 517-528 (2019).

6. Abrams, D.A., Kochalka, J., Bhide, S., Ryali, S. \& Menon, V. Intrinsic functional architecture of the human speech processing network. Cortex 129, 41-56 (2020).

7. Nourski, K.V., et al. Electrophysiology of the Human Superior Temporal Sulcus during Speech Processing. Cereb Cortex 31, 1131-1148 (2021).

8. Rauschecker, J.P. \& Scott, S.K. Maps and streams in the auditory cortex: nonhuman primates illuminate human speech processing. Nat Neurosci 12, 718-724 (2009).

9. Hickok, G. \& Poeppel, D. The cortical organization of speech processing. Nat Rev Neurosci 8, 393402 (2007).

10. Friederici, A.D. The cortical language circuit: from auditory perception to sentence comprehension. Trends Cogn Sci 16, 262-268 (2012).

11. Cloutman, L.L. Interaction between dorsal and ventral processing streams: where, when and how? Brain Lang 127, 251-263 (2013).

12. Hickok, G. \& Poeppel, D. Neural basis of speech perception. Handb Clin Neurol 129, 149-160 (2015).

13. Rauschecker, J.P. Where, When, and How: Are they all sensorimotor? Towards a unified view of the dorsal pathway in vision and audition. Cortex 98, 262-268 (2018).

14. Munoz-Lopez, M.M., Mohedano-Moriano, A. \& Insausti, R. Anatomical pathways for auditory memory in primates. Front Neuroanat 4, 129 (2010).

15. Kraus, K.S. \& Canlon, B. Neuronal connectivity and interactions between the auditory and limbic systems. Effects of noise and tinnitus. Hear Res 288, 34-46 (2012).

16. Husain, F.T. \& Schmidt, S.A. Using resting state functional connectivity to unravel networks of tinnitus. Hear Res 307, 153-162 (2014).

17. Kumar, S., et al. A Brain System for Auditory Working Memory. J Neurosci 36, 4492-4505 (2016).

18. Kumar, S., et al. Oscillatory correlates of auditory working memory examined with human electrocorticography. Neuropsychologia 150, 107691 (2021).

19. Geschwind, N. The organization of language and the brain. Science 170, 940-944 (1970).

20. Hagoort, P. The neurobiology of language beyond single-word processing. Science 366, 55-58 (2019).

21. McGettigan, C. \& Scott, S.K. Cortical asymmetries in speech perception: what's wrong, what's right and what's left? Trends Cogn Sci 16, 269-276 (2012).

22. Turkeltaub, P.E. \& Coslett, H.B. Localization of sublexical speech perception components. Brain Lang 114, 1-15 (2010).

23. Leaver, A.M. \& Rauschecker, J.P. Cortical representation of natural complex sounds: effects of acoustic features and auditory object category. J Neurosci 30, 7604-7612 (2010).

24. Eisner, F., McGettigan, C., Faulkner, A., Rosen, S. \& Scott, S.K. Inferior frontal gyrus activation predicts individual differences in perceptual learning of cochlear-implant simulations. J Neurosci 30, 7179-7186 (2010). 
25. Saur, D., et al. Ventral and dorsal pathways for language. Proc Natl Acad Sci U S A 105, 1803518040 (2008).

26. Jackson, R.L., Bajada, C.J., Rice, G.E., Cloutman, L.L. \& Lambon Ralph, M.A. An emergent functional parcellation of the temporal cortex. Neuroimage 170, 385-399 (2018).

27. Visser, M., Jefferies, E. \& Lambon Ralph, M.A. Semantic processing in the anterior temporal lobes: a meta-analysis of the functional neuroimaging literature. J Cogn Neurosci 22, 1083-1094 (2010).

28. Ralph, M.A., Jefferies, E., Patterson, K. \& Rogers, T.T. The neural and computational bases of semantic cognition. Nat Rev Neurosci 18, 42-55 (2017).

29. Hipp, J.F., Hawellek, D.J., Corbetta, M., Siegel, M. \& Engel, A.K. Large-scale cortical correlation structure of spontaneous oscillatory activity. Nat Neurosci 15, 884-890 (2012).

30. Coifman, R.R., et al. Geometric diffusions as a tool for harmonic analysis and structure definition of data: diffusion maps. Proc Natl Acad Sci U S A 102, 7426-7431 (2005).

31. Coifman, R.R. \& Hirn, M.J. Diffusion maps for changing data. Applied and Computational Harmonic Analysis 36, 79-107 (2014).

32. Margulies, D.S., et al. Situating the default-mode network along a principal gradient of macroscale cortical organization. Proc Natl Acad Sci U S A 113, 12574-12579 (2016).

33. Jones, E.G., Coulter, J.D. \& Hendry, S.H. Intracortical connectivity of architectonic fields in the somatic sensory, motor and parietal cortex of monkeys. J Comp Neurol 181, 291-347 (1978).

34. Morel, A., Garraghty, P.E. \& Kaas, J.H. Tonotopic organization, architectonic fields, and connections of auditory cortex in macaque monkeys. J Comp Neurol 335, 437-459 (1993).

35. Kaas, J.H. \& Hackett, T.A. Subdivisions of auditory cortex and processing streams in primates. Proc Natl Acad Sci USA 97, 11793-11799 (2000).

36. Kaas, J.H. \& Hackett, T.A. Subdivisions of auditory cortex and levels of processing in primates. Audiol Neurootol 3, 73-85 (1998).

37. Cavada, C., Company, T., Tejedor, J., Cruz-Rizzolo, R.J. \& Reinoso-Suarez, F. The anatomical connections of the macaque monkey orbitofrontal cortex. A review. Cereb Cortex 10, 220-242 (2000).

38. Binder, J.R., Desai, R.H., Graves, W.W. \& Conant, L.L. Where is the semantic system? A critical review and meta-analysis of 120 functional neuroimaging studies. Cereb Cortex 19, 2767-2796 (2009).

39. Humphreys, G.F., Hoffman, P., Visser, M., Binney, R.J. \& Lambon Ralph, M.A. Establishing task- and modality-dependent dissociations between the semantic and default mode networks. Proc Natl Acad Sci U S A 112, 7857-7862 (2015).

40. Jackson, R.L., Hoffman, P., Pobric, G. \& Lambon Ralph, M.A. The Semantic Network at Work and Rest: Differential Connectivity of Anterior Temporal Lobe Subregions. J Neurosci 36, 1490-1501 (2016).

41. Remedios, R., Logothetis, N.K. \& Kayser, C. An auditory region in the primate insular cortex responding preferentially to vocal communication sounds. J Neurosci 29, 1034-1045 (2009).

42. Steinschneider, M., Nourski, K.V. \& Fishman, Y.I. Representation of speech in human auditory cortex: is it special? Hear Res 305, 57-73 (2013).

43. Craig, A.D. Interoception: the sense of the physiological condition of the body. Curr Opin Neurobiol 13, 500-505 (2003).

44. Kuehn, E., Mueller, K., Lohmann, G. \& Schuetz-Bosbach, S. Interoceptive awareness changes the posterior insula functional connectivity profile. Brain Struct Funct 221, 1555-1571 (2016).

45. Bernstein, L.E. \& Liebenthal, E. Neural pathways for visual speech perception. Front Neurosci 8, 386 (2014).

46. Bullmore, E. \& Sporns, O. Complex brain networks: graph theoretical analysis of structural and functional systems. Nat.Rev.Neurosci. 10, 186-198 (2009). 
47. Knecht, S., et al. Handedness and hemispheric language dominance in healthy humans. Brain $\mathbf{1 2 3}$ Pt 12, 2512-2518 (2000).

48. Price, C.J. A review and synthesis of the first 20 years of PET and fMRI studies of heard speech, spoken language and reading. Neuroimage 62, 816-847 (2012).

49. Schirmer, A., Fox, P.M. \& Grandjean, D. On the spatial organization of sound processing in the human temporal lobe: a meta-analysis. Neuroimage 63, 137-147 (2012).

50. Chang, E.F., Raygor, K.P. \& Berger, M.S. Contemporary model of language organization: an overview for neurosurgeons. J Neurosurg 122, 250-261 (2015).

51. Ardila, A., Bernal, B. \& Rosselli, M. How Localized are Language Brain Areas? A Review of Brodmann Areas Involvement in Oral Language. Arch Clin Neuropsychol 31, 112-122 (2016).

52. Vinck, M., Oostenveld, R., van Wingerden, M., Battaglia, F. \& Pennartz, C.M. An improved index of phase-synchronization for electrophysiological data in the presence of volume-conduction, noise and sample-size bias. Neuroimage 55, 1548-1565 (2011).

53. Schaefer, A., et al. Local-Global Parcellation of the Human Cerebral Cortex from Intrinsic Functional Connectivity MRI. Cereb Cortex 28, 3095-3114 (2018).

54. Hackett, T.A., Preuss, T.M. \& Kaas, J.H. Architectonic identification of the core region in auditory cortex of macaques, chimpanzees, and humans. J Comp Neurol. 441, 197-222 (2001).

55. Hackett, T.A. Anatomic organization of the auditory cortex. Handb Clin Neurol 129, 27-53 (2015).

56. Woods, D.L., et al. Functional properties of human auditory cortical fields. Front Syst Neurosci 4, 155 (2010).

57. Barton, B., Venezia, J.H., Saberi, K., Hickok, G. \& Brewer, A.A. Orthogonal acoustic dimensions define auditory field maps in human cortex. Proc Natl Acad Sci U S A 109, 20738-20743 (2012).

58. Moerel, M., De Martino, F. \& Formisano, E. An anatomical and functional topography of human auditory cortical areas. Front Neurosci 8, 225 (2014).

59. Howard, M.A., et al. Auditory cortex on the human posterior superior temporal gyrus. J Comp Neurol. 416, 79-92 (2000).

60. Nourski, K.V., et al. Spectral organization of the human lateral superior temporal gyrus revealed by intracranial recordings. Cereb Cortex 24, 340-352 (2014).

61. Hamilton, L.S., Oganian, Y., Hall, J. \& Chang, E.F. Parallel and distributed encoding of speech across human auditory cortex. Cell 184, 4626-4639 e4613 (2021).

62. Hickok, G. The functional neuroanatomy of language. Phys Life Rev 6, 121-143 (2009).

63. Beauchamp, M.S. The social mysteries of the superior temporal sulcus. Trends Cogn Sci 19, 489-490 (2015).

64. Venezia, J.H., et al. Auditory, Visual and Audiovisual Speech Processing Streams in Superior Temporal Sulcus. Front Hum Neurosci 11, 174 (2017).

65. Zachlod, D., et al. Four new cytoarchitectonic areas surrounding the primary and early auditory cortex in human brains. Cortex 128, 1-21 (2020).

66. Belin, P., Zatorre, R.J., Lafaille, P., Ahad, P. \& Pike, B. Voice-selective areas in human auditory cortex. Nature 403, 309-312 (2000).

67. Deen, B., Koldewyn, K., Kanwisher, N. \& Saxe, R. Functional Organization of Social Perception and Cognition in the Superior Temporal Sulcus. Cereb Cortex 25, 4596-4609 (2015).

68. Wilson, S.M., Bautista, A. \& McCarron, A. Convergence of spoken and written language processing in the superior temporal sulcus. Neuroimage 171, 62-74 (2018).

69. Kahn, I., Andrews-Hanna, J.R., Vincent, J.L., Snyder, A.Z. \& Buckner, R.L. Distinct cortical anatomy linked to subregions of the medial temporal lobe revealed by intrinsic functional connectivity. $J$ Neurophysiol 100, 129-139 (2008).

70. Wang, S.F., Ritchey, M., Libby, L.A. \& Ranganath, C. Functional connectivity based parcellation of the human medial temporal lobe. Neurobiol Learn Mem 134 Pt A, 123-134 (2016). 
71. Michelmann, S., et al. Moment-by-moment tracking of naturalistic learning and its underlying hippocampo-cortical interactions. Nat Commun 12, 5394 (2021).

72. Rocchi, F., et al. Common fronto-temporal effective connectivity in humans and monkeys. Neuron 109, 852-868 e858 (2021).

73. Fruhholz, S., Trost, W. \& Kotz, S.A. The sound of emotions-Towards a unifying neural network perspective of affective sound processing. Neurosci Biobehav Rev 68, 96-110 (2016).

74. Upadhyay, J., et al. Effective and structural connectivity in the human auditory cortex. J Neurosci 28, 3341-3349 (2008).

75. Maller, J.J., et al. Revealing the Hippocampal Connectome through Super-Resolution 1150Direction Diffusion MRI. Sci Rep 9, 2418 (2019).

76. Munoz-Lopez, M., Insausti, R., Mohedano-Moriano, A., Mishkin, M. \& Saunders, R.C. Anatomical pathways for auditory memory II: information from rostral superior temporal gyrus to dorsolateral temporal pole and medial temporal cortex. Front Neurosci 9, 158 (2015).

77. Olson, I.R., McCoy, D., Klobusicky, E. \& Ross, L.A. Social cognition and the anterior temporal lobes: a review and theoretical framework. Soc Cogn Affect Neurosci 8, 123-133 (2013).

78. Mesulam, M.M. Paralimbic (mesocortical) areas. in Principles of behavioral and cognitive neurology 49-54 (Oxford University Press, New York, NY, 2000).

79. Chanes, L. \& Barrett, L.F. Redefining the Role of Limbic Areas in Cortical Processing. Trends Cogn Sci 20, 96-106 (2016).

80. Hickok, G. Computational neuroanatomy of speech production. Nat Rev Neurosci 13, 135-145 (2012).

81. Simmons, W.K. \& Martin, A. The anterior temporal lobes and the functional architecture of semantic memory. J Int Neuropsychol Soc 15, 645-649 (2009).

82. Abel, T.J., et al. Direct physiologic evidence of a heteromodal convergence region for proper naming in human left anterior temporal lobe. J Neurosci 35, 1513-1520 (2015).

83. Patterson, K., Nestor, P.J. \& Rogers, T.T. Where do you know what you know? The representation of semantic knowledge in the human brain. Nat Rev Neurosci 8, 976-987 (2007).

84. Scott, S.K., Blank, C.C., Rosen, S. \& Wise, R.J. Identification of a pathway for intelligible speech in the left temporal lobe. Brain 123 Pt 12, 2400-2406 (2000).

85. Spitsyna, G., Warren, J.E., Scott, S.K., Turkheimer, F.E. \& Wise, R.J. Converging language streams in the human temporal lobe. J Neurosci 26, 7328-7336 (2006).

86. Gorno-Tempini, M.L., et al. Cognition and anatomy in three variants of primary progressive aphasia. Ann Neurol 55, 335-346 (2004).

87. Hein, G. \& Knight, R.T. Superior temporal sulcus--It's my area: or is it? J Cogn Neurosci 20, 21252136 (2008).

88. Makris, N., et al. Delineation of the middle longitudinal fascicle in humans: a quantitative, in vivo, DT-MRI study. Cereb Cortex 19, 777-785 (2009).

89. Binney, R.J., Parker, G.J. \& Lambon Ralph, M.A. Convergent connectivity and graded specialization in the rostral human temporal lobe as revealed by diffusion-weighted imaging probabilistic tractography. J Cogn Neurosci 24, 1998-2014 (2012).

90. de Heer, W.A., Huth, A.G., Griffiths, T.L., Gallant, J.L. \& Theunissen, F.E. The Hierarchical Cortical Organization of Human Speech Processing. J Neurosci 37, 6539-6557 (2017).

91. Binder, J.R., et al. Human temporal lobe activation by speech and nonspeech sounds. Cereb Cortex 10, 512-528 (2000).

92. Cogan, G.B., et al. Sensory-motor transformations for speech occur bilaterally. Nature 507, $94-98$ (2014).

93. Gonzalez Alam, T., et al. A tale of two gradients: differences between the left and right hemispheres predict semantic cognition. Brain Struct Funct (2021). 
94. Karolis, V.R., Corbetta, M. \& Thiebaut de Schotten, M. The architecture of functional lateralisation and its relationship to callosal connectivity in the human brain. Nat Commun 10, 1417 (2019).

95. Sherman, S.M. \& Guillery, R.W. Distinct functions for direct and transthalamic corticocortical connections. J Neurophysiol 106, 1068-1077 (2011).

96. Hu, B. Functional organization of lemniscal and nonlemniscal auditory thalamus. Exp Br Res 153, 543-549 (2003).

97. Biswal, B.B., et al. Toward discovery science of human brain function. Proc Natl Acad Sci U S A 107, 4734-4739 (2010).

98. Seitzman, B.A., Snyder, A.Z., Leuthardt, E.C. \& Shimony, J.S. The State of Resting State Networks. Top Magn Reson Imaging 28, 189-196 (2019).

99. Nourski, K.V. \& Howard, M.A., 3rd. Invasive recordings in the human auditory cortex. Handb Clin Neurol 129, 225-244 (2015).

100. Jenkinson, M., Bannister, P., Brady, M. \& Smith, S. Improved optimization for the robust and accurate linear registration and motion correction of brain images. Neuroimage 17, 825-841 (2002).

101. Rohr, K., et al. Landmark-based elastic registration using approximating thin-plate splines. IEEE Trans Med Imaging 20, 526-534 (2001).

102. Destrieux, C., Fischl, B., Dale, A. \& Halgren, E. Automatic parcellation of human cortical gyri and sulci using standard anatomical nomenclature. Neuroimage 53, 1-15 (2010).

103. Destrieux, C., et al. A practical guide for the identification of major sulcogyral structures of the human cortex. Brain Struct Funct 222, 2001-2015 (2017).

104. Brugge, J.F., et al. Coding of repetitive transients by auditory cortex on Heschl's gyrus. $J$ Neurophysiol 102, 2358-2374 (2009).

105. Nourski, K.V., Steinschneider, M. \& Rhone, A.E. Electrocorticographic Activation within Human Auditory Cortex during Dialog-Based Language and Cognitive Testing. Front Hum Neurosci 10, 202 (2016).

106. Power, J.D., Barnes, K.A., Snyder, A.Z., Schlaggar, B.L. \& Petersen, S.E. Spurious but systematic correlations in functional connectivity MRI networks arise from subject motion. Neuroimage 59, 2142-2154 (2012).

107. Kovach, C.K. \& Gander, P.E. The demodulated band transform. J Neurosci Methods 261, 135-154 (2016).

108. Banks, M.I., et al. Cortical functional connectivity indexes arousal state during sleep and anesthesia. Neuroimage 211, 116627 (2020).

109. Langs, G., Golland, P., Tie, Y., Rigolo, L. \& Golby, A.J. Functional Geometry Alignment and Localization of Brain Areas. Adv Neural Inf Process Syst 1, 1225-1233 (2010).

110. Satopaa, V., Albrecht, J., Irwin, D. \& Raghavan, B. Finding a "Kneedle" in a Haystack: Detecting Knee Points in System Behavior. in 2011 31st International Conference on Distributed Computing Systems Workshops 166-171 (2011). 